\title{
A Stochastic Frontier Model with Structural Breaks in Efficiency and Technology
}

\author{
Guangjie LI \\ ligj@cf.ac.uk \\ Cardiff Business School \\ Cardiff University
}

February 2010

\begin{abstract}
We develop a stochastic frontier model with unknown locations and number of structural breaks for both efficiency and technology. The structural changes in technology and efficiency are different. It is possible to identify these two types of changes even under a short panel context. The algorithm to estimate the model is designed to avoid the potential pitfalls with panel data. Parallel computing is used to tackle the estimation obstacles. We demonstrate the viability of our method with artificial data and we also apply it to study the world production over the period between 1960 and 2007.
\end{abstract}

JEL Classification Code: C11, C13, C15, C63, O0

Keywords: stochastic frontier model, panel data, change point model, hidden Markov model, Markov chain Monte Carlo, parallel computing

\section{Introduction}

Stochastic frontier model used to study either production or cost efficiency was introduced by Aigner et al. (1977) and Meeusen and van den Broeck (1990). Under panel data, a key focus of the model is to estimate the efficiency levels for different individuals. Usually the efficiency terms are assumed to be heterogenous across individuals. From an econometric point of view, the number of parameters to be estimated in the model, i.e. individual efficiencies, will increase with the cross section dimension and it is generally difficult to estimate such parameters. If individual efficiencies are constant over time, we can better estimate them with longer time span. However, long panel is seldom the norm in practice. Moreover, even if long panel is present, it is hard to believe that efficiencies and the technology employed in the production are time-invariant. There have been some attempts to model such time varying behaviour of efficiencies and technology. Tsionas (2006) used an AR process to model the 
persistency and temporal changes of individual efficiencies. Tsionas and Kumbhakar (2004) assumed there are a fixed number of regimes in both technology and efficiency which have a Markov switching structure as in Hamilton (1989) and Hamilton (1990). Tsionas and Tran (2007) developed a threshold stochastic frontier model and the regime switching is controlled by whether an observed variable crosses a threshold value.

For the change point model literature, it has been advocated that the number of change-points should be treated as unknown, see e.g. Koop and Potter (2009) and Giordani and Kohn (2008). Such methods are more flexible and practical than the methods that require to prespecify the number of regimes a priori. We apply these methods to stochastic frontier model and let the efficiency and technology have unknown number of regimes and unknown location of break points, which will be determined by the data. Another thing different from the previous literature in this paper is that we allow efficiency and technology to have different change-points. It should be sensible to assume that efficiency and technology may not change hand in hand. On the one hand, a firm's efficiency may be improved due to better management or other factors unrelated to technology breakthrough. On the other hand, even if a firm adopts a new technology, it is not certain that the firm's efficiency will improve as a result. In the growth literature, technological change and technological catch-up are identified as two sources of economic growth (see Kumar and Russell, 2002). When there is adoption of new technology, the production frontier should be shifted upward. However, this does not necessarily imply that some firms will make better use of the new technology than the others. While when there is an improvement in efficiency, the less well-performing individuals are expected to catch up with their better performing peers even though the production frontier of the whole society remains constant. For the structural break in efficiency, the change can be two folds. It is possible that either the overall level of efficiency will vary, or the firms will exhibit convergence or divergence in efficiency, or both may happen at the same time. We will consider all of these in our model by allowing the log of the efficiency term to follow a log normal distribution, which will also make our paper different from previous work to only look into changes in dispersion of efficiency.

The plan of the paper will be as follows. Section 2 describes the model and the algorithm to estimate it. Section 3 gives an example using artificially generated data. Section 4 applies the method to study the world production over the period between 1960 and 2007. Finally Section 5 concludes.

\section{The Model}

Suppose we are dealing with production stochastic frontier. Our model takes the following form:

$$
\begin{aligned}
y_{i t} & =x_{i t}^{\prime} a_{S_{t}^{a}}+w_{i t}^{\prime} \beta-\exp \left(\gamma_{i S_{t}^{\gamma}}\right)+\epsilon_{i t}, \\
\epsilon_{i t} & \sim i . i . d . N\left(0, \sigma^{2}\right), \quad i=1, \ldots, N, t=1, \ldots, T
\end{aligned}
$$


where $y$ is the log output and $(x, w)$ is a collection of the log inputs and the intercept. The inputs are assumed to be exogenous and are separated into $x_{i t}$ and $w_{i t}$, which correspond to the regime-changing parameter $a$ and the constant $\beta$ respectively ${ }^{1}$. The production frontier is given by $x^{\prime} a+w^{\prime} \beta$ and $\epsilon$ is the disturbance or measurement error which makes the frontier stochastic. The technical efficiency is calculated by $\tau=\exp [-\exp (\gamma)]$ with $\tau=1$ being the most efficient. We adopt a hierarchical prior for $\gamma$ :

$$
\gamma_{i, S_{t}^{\gamma}=j} \sim N\left(\mu_{j}, \exp \left(\phi_{j}\right)\right) .
$$

which implies that $\exp (\gamma)$ follows a $\log$ normal distribution. Such specification can also be seen in Tsionas (2006) and Yan et al. (2009). Compared to the half normal specification (e.g. Tsionas and Kumbhakar, 2004) and the exponential distribution (e.g. van den Broeck et al., 1994), such prior is more flexible and can have a bell shape. It is possible to consider both changes in level and changes in volatility for efficiency under such assumption. Moreover it is convenient to develop algorithms for estimation under unknown number of structural breaks as will be explained later. We assume that the value of $a$ and the distribution of $\gamma$ may change over time and attach a regime state index $S_{t}$ to each of them, $S_{t}=1, \ldots, M . M$ is the maximal number of regimes in sample. Note that in such setup, under the same regime, $\gamma$ is the same for an individual across different periods. It should be a sensible assumption that individual's efficiency may remain the same over a certain period of time, but may have structural changes given a long period. In addition, the longer time span $\gamma$ remains the same, the more reliable estimates can we obtain. If we could correctly separate the data into different regimes, it should help us better estimate an individual's efficiency score. The two processes $S_{t}^{a}$ and $S_{t}^{\gamma}$ are independent of each other. Following Chib (1998), we assume that they are Markovian, i.e. the conditional probability of the current state only depends on the previous state and the current state is equal to either the previous state or the previous state plus one with the initial state $S_{1}=1$. Koop and Potter (2007) and Koop and Potter (2009) noted that imposing the number of in-sample regimes a priori implies a prior under which breaks near the end of the sample is unusually more likely than the previous periods. They argued that one should treat the number of in-sample regimes as unknown to avoid this problem. By setting $M=T$, we actually imply that the number of regimes ranging from 1 to $T$ inside our sample are all possible and will be determined by the data. Koop and Potter (2009) proposed to extend the breakpoints to take place outside the sample and use a uniform prior on the location of the break points. Their prior can take the following form:

$$
\operatorname{Pr}\left(S_{t+1}=m+1 \mid S_{t}=m, d_{t, m}\right)=\left\{\begin{array}{l}
0 \quad \text { if } m>t \\
\frac{1}{D-d_{t, m}+1} \quad \text { if } m \leq t, \text { for } d_{t, m} \leq D,
\end{array}\right.
$$

\footnotetext{
${ }^{1}$ We can assume the intercept is either constant or time-varying. In other words, it can be an element in either $a$ or $\beta$.
} 
where $m=1, \ldots, M, D$ is the maximal duration of one regime, $d_{t, m}$ denotes the duration of current regime $m$ at period $t$ with $d_{t, m}=1, \ldots, t-m+1$, $\operatorname{Pr}\left(S_{t+1}=m \mid S_{t}=m, d_{t, m}\right)=1-\operatorname{Pr}\left(S_{t+1}=m+1 \mid S_{t}=m, d_{t, m}\right)$ and $\operatorname{Pr}\left(S_{1}=\right.$ $1)=1$. Koop and Potter (2009) also suggested to treat $M$ and $D$ as unknown parameters to be estimated. In our example and application below, we simply fix $M=D=T$, which allows for the cases from only one regime to $T$ regimes ${ }^{2}$ in sample. The prior proposed by Koop and Potter (2009) implies a flat prior for $d_{t, m}$ over its support and hence the Markov transition probabilities of the states unconditional on $d_{t, m}$ can be calculated by

$$
\operatorname{Pr}_{m j}=\operatorname{Pr}\left(S_{t+1}=j \mid S_{t}=m\right)=\sum_{d_{t, m}=1}^{t-m+1} \frac{1}{t-m+1} \operatorname{Pr}\left(S_{t+1}=j \mid S_{t}=m, d_{t, m}\right) .
$$

where $j$ is equal to either $m$ or $m+1$. By rewriting the prior proposed by Koop and Potter (2009), we can easily use the algorithm developed by Chib (1998) for our estimation.

We assume both the value of $a$ and the parameters for the distribution of $\gamma$ will change over time. Below are the processes they follow:

$$
\begin{array}{ll}
\mu_{S_{t}^{\gamma}}=\mu_{S_{t-1}^{\gamma}}+u_{S_{t}^{\gamma}}^{1}, & u_{S_{t}^{\gamma}}^{1} \sim \text { i.i.d.N }\left(0, \sigma_{\mu}^{2}\right), \\
\phi_{S_{t}^{\gamma}}=\phi_{S_{t-1}^{\gamma}}+u_{S_{t}^{\gamma}}^{2}, & u_{S_{t}^{\gamma}}^{2} \sim i . i . d . N\left(0, \sigma_{\phi}^{2}\right) . \\
a_{S_{t}^{a}}=a_{S_{t-1}^{a}}+u_{S_{t}^{a}}, & u_{S_{t}^{a}} \sim \text { i.i.d.N }\left(0, \sigma_{a}^{2} I\right)
\end{array}
$$

which means that different regimes of $a$ and the distribution parameters for $\gamma$ are all connected by random walk. Note that when there is a structural break in $\gamma$, both its mean and variance will change. It is easy to allow the mean and the variance for efficiency to have different break points. The elements of $a$ can also have similar treatments. All of these just require more computation under our framework.

Equation (5) and (6) can be viewed as the priors for the difference between the parameters under two regimes. In what follows, we just need to specify the priors for the initial regime parameters: $a_{1}, \mu_{1}$ and $\phi_{1}$, which are all flat priors. To summarize, the following are the priors used in our Bayesian analysis:

$$
\begin{aligned}
& p\left(a_{1}\right), p\left(\mu_{1}\right), p\left(\phi_{1}\right) \propto 1 \\
& \sigma_{a}^{2} \sim I G\left(\zeta_{a}, v_{a}\right) ; \sigma_{\mu}^{2} \sim \operatorname{IG}\left(\zeta_{\mu}, v_{\mu}\right) ; \sigma_{\phi}^{2} \sim \operatorname{IG}\left(\zeta_{\phi}, v_{\phi}\right) \\
& p\left(\sigma^{2}\right) \propto \frac{1}{\sigma^{2}} \\
& \beta \mid \sigma^{2} \sim I(\beta>0) N\left(0, \sigma^{2}\left(g \sum_{i=1}^{N} \sum_{t=1}^{T} x_{i t} x_{i t}^{\prime}\right)^{-1}\right)
\end{aligned}
$$

\footnotetext{
${ }^{2}$ When the number of regimes equal to the number of periods, it is the time-varying parameter (TVP) model.
} 
where $I G$ denotes the inverted gamma distribution with mean $\frac{\zeta}{v-2}$ and degrees of freedom $v . g$ is the parameter controlling the informativeness of the g-prior (see Zellner, 1986), which is chosen to be $\frac{1}{N T}$. Such choice of $g$ ensures consistency in model selection (see Fernandez et al., 2001). The prior of $\beta$ is a normal distribution truncated to the positive region since $\beta$ are the elasticity parameters in the production function. We choose $v=2$ and $\zeta=4$ to ensure the values of $a, \mu$ and $\phi$ can wander a wide range. To see this, note that for the inverted gamma distribution with $v=2$ the first moment does not exist. Hence it is possible for $\sigma_{a}^{2}, \sigma_{\mu}^{2}$ and $\sigma_{\phi}^{2}$ to take quite large values and parameters under different regimes could be quite different from each other.

We now turn to the posterior simulation of the regimes and parameters.

Algorithm 2.1. The following are the steps to simulate the regime states and the parameters from their posterior:

1. draw $S^{\gamma} \mid \mu, \phi, a, S^{a}, \beta, \sigma^{2}, Y$ and $\gamma \mid S^{\gamma}, \mu, \phi, a, S^{a}, \beta, \sigma^{2}, Y$;

2. draw $\sigma_{\mu}^{2} \mid S^{\gamma}, \phi, \gamma, Y$ and $\mu \mid \sigma_{\mu}^{2}, S^{\gamma}, \phi, \gamma, Y$;

3. $d r a w \sigma_{\phi}^{2} \mid S^{\gamma}, \mu, \gamma, Y$ and $\phi \mid \sigma_{\phi}^{2}, S^{\gamma}, \mu, \gamma, Y$;

4. draw $S^{a}\left|\sigma_{a}^{2}, \gamma, \beta, \sigma^{2}, Y, \sigma_{a}^{2}\right| S^{a}, \gamma, \beta, \sigma^{2}, Y$ and $a \mid \sigma_{a}^{2}, S^{a}, \gamma, \beta, \sigma^{2}, Y$;

5. draw

$$
\begin{aligned}
& \sigma^{2} \mid a, \gamma, Y \sim I G\left(\sum_{i=1}^{N} \sum_{t=1}^{T}\left[y_{i t}-x_{i t}^{\prime} a_{S_{t}^{a}}+\exp \left(\gamma_{i S_{t}^{\gamma}}\right)\right]^{2}\right. \\
& -\frac{1}{1+g}\left\{\sum_{i=1}^{N} \sum_{t=1}^{T}\left[y_{i t}-x_{i t}^{\prime} a_{S_{t}^{a}}+\exp \left(\gamma_{i S_{t}^{\gamma}}\right)\right] w_{i t}\right\}^{\prime} \\
& \left.\left(\sum_{i=1}^{N} \sum_{t=1}^{T} w_{i t} w_{i t}^{\prime}\right)^{-1}\left\{\sum_{i=1}^{N} \sum_{t=1}^{T}\left[y_{i t}-x_{i t}^{\prime} a_{S_{t}^{a}}+\exp \left(\gamma_{i S_{t}^{\gamma}}\right)\right] w_{i t}\right\}, N T\right)
\end{aligned}
$$

6. draw

$$
\begin{aligned}
& \beta \mid \sigma^{2}, a, \gamma, Y \sim \\
& I(\beta>0) N\left(\frac{1}{1+g}\left[\sum_{i=1}^{N} \sum_{t=1}^{T} x_{i t} w_{i t}^{\prime}\right]^{-1}\left\{\sum_{i=1}^{N} \sum_{t=1}^{T}\left[y_{i t}-x_{i t}^{\prime} a_{S_{t}^{a}}+\exp \left(\gamma_{i S_{t}^{\gamma}}\right)\right] w_{i t}\right\}\right. \\
& \left.\sigma^{2}\left[(1+g) \sum_{i=1}^{N} \sum_{t=1}^{T} w_{i t} w_{i t}^{\prime}\right]^{-1}\right) .
\end{aligned}
$$

where $S=\left(S_{1}, \ldots, S_{T}\right)^{\prime}$ and $a, \mu$ and $\phi$ consist of $M$ regimes. 
Algorithm 2.1 separate the parameters into blocks and use Gibbs sampler to draw them from their conditional posteriors. More details of the algorithm can be found in the appendix. Basically, we have $S^{\gamma}$ and $\gamma, \sigma_{a}^{2}, S^{a}$ and $a, \sigma_{\mu}^{2}$ and $\mu, \sigma_{\phi}^{2}$ and $\phi, \sigma^{2}$ and $\beta$ as one block respectively. For some steps, given a group of parameters, other parameters will be irrelevant in the conditional posterior.

To draw the states of the regime, we use two different algorithms for $S^{\gamma}$ and $S^{a}$. Drawing $S^{\gamma}$ involves integrating out $\gamma$ and the conditional posterior depends on the values of $\mu$ and $\phi$ of different regimes. Since the conditional posterior of $\gamma$ is not a standard distribution, its integrating constant does not have closed form and $\gamma$ has to be integrated out numerically. We use adaptive quadrature to obtain the integrating constant, which involves substantial computation cost. Fortunately this problem can be parallelized and the computation is done by ARCCA Merlin, which is a computing cluster based in Cardiff University. The basic idea of parallel computing is to split a big computing job into smaller jobs and make them run on many computer CPUs at the same time. To simulate a draw of $S^{\gamma}$ requires finding the integrating constant for the posterior conditional of $\gamma$, which is an $N \times 1$ vector. As is explained in the appendix, this demands $\frac{(T-1)(T+2) N}{2}$ integrating operations. Since we are using a prior with different $\gamma_{i} \mathrm{~s}$ independent of each other, the integration with respect to different $\gamma_{i} \mathrm{~s}$ is not related with each other so that we can spread out the computation task across the cluster to speed up the computation. The problem of drawing from the posterior of $\gamma$ conditional on other parameters is similar. Although the conditional distribution is not a standard one, we can simulate different $\gamma_{i} \mathrm{~S}$ separately at the same time by inverting its posterior cumulative distribution function. All details can be found in the appendix.

When we draw $S^{\gamma}$, we mainly follow the algorithm proposed by Chib (1998) (see also Koop and Potter, 2007) and the conditional distribution of the regime states depend on the values of the parameters, ie. $\mu$ and $\phi$, of different regimes. Gerlach et al. (2000) pointed out that such algorithm relying on the regime parameters could be very inefficient when the regime states and the regime parameters are highly correlated and the Markov chain could get stuck in a regime for too long. Under the panel context of ours, when we draw $a$ and its regime states, this problem can be very serious. To see this problem, consider the following example:

$$
\begin{aligned}
y_{i t} & =a_{S_{t}}+\epsilon_{i t}, \quad \epsilon_{i t} \sim \text { i.i.d.N }\left(0, \sigma^{2}\right), \\
a_{S_{t}} & =a_{S_{t-1}}+u_{S_{t}}, \quad u_{S_{t}} \sim \text { i.i.d.N }\left(0, \sigma_{a}^{2}\right) .
\end{aligned}
$$

The above model is just a simplified version of Equation (1) without the exogenous regressors and the inefficiency terms. We first use the algorithm proposed by Chib (1998) and let the posterior of the regime states $\left(S_{t}\right)$ conditional on the regime parameters $(a)$ during the posterior simulation. The prior specification is the same as mentioned before. We set $N=100, T=4$ and $\sigma^{2}=1$ to generate artificial data. The break point is set to $T=2^{3}$ with $a_{1}=0$ and $a_{2}=1$. When

\footnotetext{
${ }^{3}$ That is period 1 and 2 are under the same regime and period 3 and 4 constitute another regime.
} 
we initialize the Markov chain, we set the initial draw for the regime states as $(1,2,3,3)^{\prime}$ and the regime parameter values as $(0,0,1)$ with the parameter of the last regime, which takes place outside the sample, determined by the prior in (10). For this initial draw, we set the number of in-sample regimes to be 3 and the first two break points to be period 1 and 2 . Results after $10^{5}$ draws are shown in Table 1, 2 and 3 with the corresponding results indicated by "Chib" or "C". For comparison, we have also put down the probabilities implied by the prior in (3) and (4). We can see that the Markov chain is stuck in the initial state throughout the simulation, that is, the first two break points are always period one and two, which makes the posterior probability of the number of regimes being 3 abnormally high. The reason for this can be explained as follows. When we draw $a$ conditional on its states, the bigger $N$ is, the tighter the posterior distribution of $a$ in different regimes will become. It will make the values of different regimes less likely to overlap each other. This can be confirmed from Table 4 . The posterior intervals of $a_{2}$ and $a_{3}$ have no overlapping sections such that $y_{i 3}$ will never switch to state 2 during the simulation since $a_{3}$ is always closer to the true value than $a_{2}$.

\begin{tabular}{|l||c|c|c|c|}
\hline regime no. & 1 & 2 & 3 & 4 \\
\hline prior & 0.2500000 & 0.5598958 & 0.1744792 & 0.0156250 \\
Chib & 0.0000000 & 0.0000000 & 0.9674400 & 0.0325600 \\
Gerlach & 0.0000000 & 0.5998200 & 0.3873300 & 0.0128500 \\
\hline
\end{tabular}

Table 1: The number of regime(s) probabilities

\begin{tabular}{|l||c|c|c|}
\hline period & 1 & 2 & 3 \\
\hline prior & 0.2500000 & 0.3125000 & 0.3932292 \\
Chib & 1.0000000 & 1.0000000 & 0.0325600 \\
Gerlach & 0.3761600 & 1.0000000 & 0.0368700 \\
\hline
\end{tabular}

Table 2: The probability of period t being a break point

To get around this problem for partially Gaussian state space models, Gerlach et al. (2000) suggested to draw the regime states unconditional on the regime parameters ${ }^{4}$. To use their algorithm, we have to rewrite the process of $a$ in Equation (10) as

$$
a_{t+1}=a_{t}+K_{t} u_{t}, \quad u_{t} \sim \text { i.i.d.N }\left(0, \sigma_{a}^{2}\right) .
$$

\footnotetext{
${ }^{4}$ The author acknowledges that the aforementioned problem of drawing the regime states conditional on the regime parameters may also exist when drawing $S^{\gamma}$. But unlike drawing the intercept, the simulation problem can not be changed into a Gaussian state space problem. To the best knowledge of the author, the algorithm by Chib (1998) is the only feasible algorithm in this respect.
} 


\begin{tabular}{|l||c|c|c|c|}
\hline period & not exist & 1 & 2 & 3 \\
\hline breakpoint 1(p) & 0.2500000 & 0.2500000 & 0.2500000 & 0.2500000 \\
breakpoint 2(p) & 0.8098958 & 0.0000000 & 0.0625000 & 0.1276042 \\
breakpoint 3(p) & 0.9843750 & 0.0000000 & 0.0000000 & 0.0156250 \\
breakpoint 1(C) & 0.0000000 & 1.0000000 & 0.0000000 & 0.0000000 \\
breakpoint 2(C) & 0.0000000 & 0.0000000 & 1.0000000 & 0.0000000 \\
breakpoint 3(C) & 0.9674400 & 0.0000000 & 0.0000000 & 0.0325600 \\
breakpoint 1(G) & 0.0000000 & 0.3761600 & 0.6238400 & 0.0000000 \\
breakpoint 2(G) & 0.5998200 & 0.0000000 & 0.3761600 & 0.0240200 \\
breakpoint 3(G) & 0.9871500 & 0.0000000 & 0.0000000 & 0.0128500 \\
\hline
\end{tabular}

Table 3: The probabilities of breakpoints at period $t$

\begin{tabular}{|l||c|r|r|r|r|c|}
\hline & $\sigma^{2}$ & \multicolumn{1}{|c|}{$a_{1}$} & \multicolumn{1}{c|}{$a_{2}$} & \multicolumn{1}{c|}{$a_{3}$} & \multicolumn{1}{c|}{$a_{4}$} & $\sigma_{a}^{2}$ \\
\hline $0 \%$ & 0.6868 & -0.4392 & -0.7708 & 0.6684 & -25.0576 & 0.2069 \\
$25 \%$ & 0.8836 & -0.0592 & -0.4023 & 0.9640 & 0.1197 & 1.1647 \\
$50 \%$ & 0.9267 & 0.0048 & -0.3372 & 1.0100 & 1.0067 & 1.8641 \\
$75 \%$ & 0.9721 & 0.0701 & -0.2732 & 1.0563 & 1.9073 & 3.2257 \\
$100 \%$ & 1.2956 & 0.4401 & 0.1382 & 1.3404 & 29.2113 & 246.1007 \\
\hline
\end{tabular}

Table 4: Posterior quantiles using Chib's Algorithm

\begin{tabular}{|l||c|c|r|r|r|c|}
\hline & $\sigma^{2}$ & \multicolumn{1}{|c|}{$a_{1}$} & \multicolumn{1}{c|}{$a_{2}$} & \multicolumn{1}{c|}{$a_{3}$} & \multicolumn{1}{c|}{$a_{4}$} & $\sigma_{a}^{2}$ \\
\hline $0 \%$ & 0.6907 & -0.4547 & -0.7533 & -42.9638 & -62.6465 & 0.2011 \\
$25 \%$ & 0.8910 & -0.1870 & -0.2964 & 0.7077 & -0.1788 & 1.2585 \\
$50 \%$ & 0.9346 & -0.1219 & 0.9521 & 1.0096 & 1.0184 & 2.1070 \\
$75 \%$ & 0.9811 & -0.0255 & 1.0283 & 1.3314 & 2.2087 & 3.8758 \\
$100 \%$ & 1.2922 & 0.4020 & 1.4238 & 33.8063 & 45.0242 & 811.4638 \\
\hline
\end{tabular}

Table 5: Posterior quantiles using the algorithm by Gerlach et al. 
where $K_{t}=S_{t+1}-S_{t}$ takes the value of either 1 or 0 indicating whether period $t$ is a break point or not. If $S_{t}$ follows a Markov process as in (3), the prior of $K$ will become

$$
\begin{aligned}
\operatorname{Pr}(K) & =\operatorname{Pr}\left(K_{T-1} \mid K_{2:(T-2)}\right) \ldots \operatorname{Pr}\left(K_{1}\right) \\
& =\operatorname{Pr}\left(S_{T} \mid S_{T-1}\right) \operatorname{Pr}\left(S_{T-1} \mid S_{T-2}\right) \ldots \operatorname{Pr}\left(S_{2} \mid S_{1}\right)
\end{aligned}
$$

where $K_{i: j}$ denotes $K_{i}, \ldots, K_{j}$. The details of the algorithm adapted from Gerlach et al. (2000) are given in the appendix. The previous tables also show the results obtained from this algorithm given the same initial draw as before. We can see that the Markov chain now does not get stuck in the initial draw of the regime state and the true regime state with period 2 as the first and the only break point has the highest level of posterior probability. Table 5 shows the posterior quantiles for the parameters obtained from this algorithm, which are all within reasonable ranges.

\section{An example using artificial data}

In this section we give an example with artificially generated data. The model is given in (1). We set $T=4$ and $N=100$. There are two regimes for both $\gamma$ and $a$. There is only one input (exogenous regressor) and $a_{i t}$ is a scalar. In other words, $x_{i t}$ is a vector of ones and only the intercept in (1) changes over time while the slope remains constant. The break point for $\gamma$ is at period 2 and $a$, whose values for the two regimes are 1 and 2 respectively, has its breakpoint at period 3. The structural parameters that generate $\gamma$ are set as $\mu=(0,-1)^{\prime}$ and $\phi=(0,0)^{\prime}$, which implies from regime 1 to regime 2 , the median of the technical efficiency $\tau=\exp [-\exp (\gamma)]$ increases from around $37 \%$ to $70 \%$ and its standard deviation decreases from about $26.6 \%$ to $24 \%$. We generate $\exp \left(w_{i t}\right)$ (only one input) from a uniform distribution with minimum as 1 and maximum as $20, \beta=2$ and $\sigma^{2}=0.3$.

The plots of the data are shown in Figure 1. The regime patterns may not be very obvious from the graph. The regime estimation results shown in Table 6, 7 and $8^{5}$ are obtained after 20,000 draws with 1,000 burn-in. For the regime inference, we can see that the posterior results are in stark contrast with the prior listed in Table 1, 2 and 3. It is clear that Algorithm 2.1 can correctly identify the number of regimes and the location of the break points for both $\gamma$ and $a$ with the correct values receiving the highest posterior probabilities.

The posterior point estimates and quantile estimates are shown in Table 9 and Table 10 respectively with $S t d$ denoting standard deviation and NSE for numerical standard error ${ }^{6}$. Note that when we use different ways to separate

\footnotetext{
${ }^{5}$ The letter "e" inside the brackects indicates that those are the results for efficiency and "t" for technology.

${ }^{6}$ The naive NSE is just the standard deviation divided by the square root of number of draws while time series NSE takes into account the serial correlation of the draws (see Plummer et al., 2009).
} 


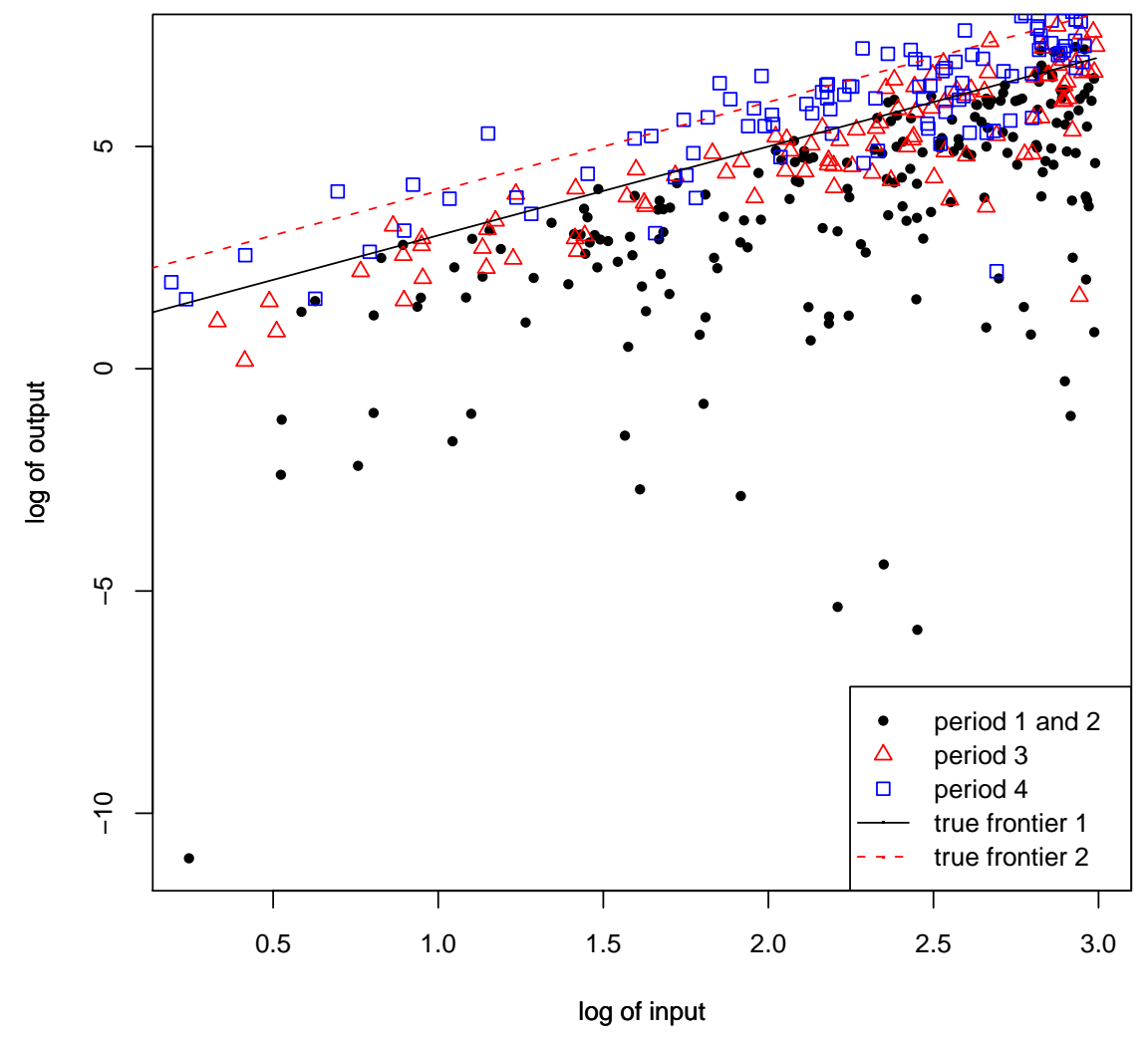

Figure 1: Plot of the artificial output and input in log

\begin{tabular}{|l||c|c|c|c|}
\hline regime no. & 1 & 2 & 3 & 4 \\
\hline efficiency & 0.00000 & 0.58850 & 0.38660 & 0.02490 \\
technology & 0.00000 & 0.79190 & 0.20315 & 0.00495 \\
\hline
\end{tabular}

Table 6: The number of regime(s) probabilities

\begin{tabular}{|l||c|c|c|}
\hline period & 1 & 2 & 3 \\
\hline efficiency & 0.37785 & 1.00000 & 0.05855 \\
technology & 0.03625 & 0.17680 & 1.00000 \\
\hline
\end{tabular}

Table 7: The probability of period t being a break point 


\begin{tabular}{|l||c|c|c|c|}
\hline period & not exist & 1 & 2 & 3 \\
\hline breakpoint 1(e) & 0.00000 & 0.37785 & 0.62215 & 0.00000 \\
breakpoint 2(e) & 0.58850 & 0.00000 & 0.37785 & 0.03365 \\
breakpoint 3(e) & 0.97510 & 0.00000 & 0.00000 & 0.02490 \\
breakpoint 1(t) & 0.00000 & 0.03625 & 0.17185 & 0.79190 \\
breakpoint 2(t) & 0.79190 & 0.00000 & 0.00495 & 0.20315 \\
breakpoint 3(t) & 0.99505 & 0.00000 & 0.00000 & 0.00495 \\
\hline
\end{tabular}

Table 8: The probability of breakpoint at period $t$

the data into regimes based on the regime states and estimate the regime parameters, we are virtually doing model averaging. The parameters that are not common to different model specifications may be multi-modal and may not have bell shapes. For such cases, the posterior standard deviation would not be very informative. This is why we also list the posterior quantile results. All estimates are within sensible ranges. Figure 2 compares the efficiency estimates based on the posterior median to the true individual efficiencies. We can see that the estimates for period 1 and period 2 are close to each other while period 3 and period 4 are almost identical. These are due to the regime state estimation results, which suggest the break point is most likely to appear at period 2 and there should be two regimes. Our algorithm has no difficulty to identify the two regimes for efficiency. However, the estimated density plots are quite different from the true ones. Note that for each regime, the maximal duration is just two periods in our simulation, which means we only have two observations to estimate each individual efficiency and there are $2 N$ such parameters. We could expect that as the duration for each regime gets bigger, our estimates for the individual efficiencies will become closer to the true values.

\section{An empirical example}

We use the dataset available from Penn World Table Index 6.3 (see Heston et al., 2009) for our empirical application. The previous verions of this dataset have been used in Kumar and Russell (2002) and Tsionas and Kumbhakar (2004). The key focuses of the study are to investigate the changes of the production frontier of the world and the production efficiencies of different countries over time and their relationship with economic growth. We have collected the data of $110^{7}$ countries or regions over the period from 1960 to 2007. The basic model is the same as (1), where output is measured by gross domestic product ( rgdpch $\times$ $P O P)$ and the inputs are capital $(r g d p l \times P O P \times k i / 100)$ and labour $(r g d p c h \times$ $P O P /$ rgdpwok). Both output and inputs enter the equation as logs. To smooth out business cycles and also to keep the computation time manageable, we

\footnotetext{
${ }^{7}$ We only keep those countries and regions with all the variables available duirng 1960-2007. There are two versions of China in the dataset, which are not used in our following analysis.
} 


\begin{tabular}{|l||c|c|c|c|}
\hline & Mean & Std & Naive NSE & Time-series NSE \\
\hline$\sigma^{2}$ & 0.399 & 0.059 & 0.000 & 0.002 \\
$\beta$ & 2.004 & 0.058 & 0.000 & 0.003 \\
$\mu_{1}$ & -0.049 & 0.272 & 0.002 & 0.020 \\
$\mu_{2}$ & -1.168 & 0.983 & 0.007 & 0.115 \\
$\mu_{3}$ & -1.790 & 2.005 & 0.014 & 0.056 \\
$\mu_{4}$ & -1.777 & 2.915 & 0.021 & 0.058 \\
$\phi_{1}$ & 0.175 & 0.339 & 0.002 & 0.023 \\
$\phi_{2}$ & 0.379 & 0.480 & 0.003 & 0.039 \\
$\phi_{3}$ & 0.441 & 1.172 & 0.008 & 0.041 \\
$\phi_{4}$ & 0.438 & 1.670 & 0.012 & 0.041 \\
$a_{1}$ & 0.853 & 0.207 & 0.001 & 0.016 \\
$a_{2}$ & 1.633 & 0.375 & 0.003 & 0.022 \\
$a_{3}$ & 1.804 & 1.899 & 0.013 & 0.017 \\
$a_{4}$ & 1.770 & 2.788 & 0.020 & 0.023 \\
\hline
\end{tabular}

Table 9: Posterior point estimates

\begin{tabular}{|l||r|r|r|r|c|}
\hline & \multicolumn{1}{|c|}{$2.5 \%$} & \multicolumn{1}{c|}{$25 \%$} & \multicolumn{1}{c|}{$50 \%$} & \multicolumn{1}{c|}{$75 \%$} & $97.5 \%$ \\
\hline$\sigma^{2}$ & 0.323 & 0.367 & 0.395 & 0.425 & 0.500 \\
$\beta$ & 1.886 & 1.966 & 2.006 & 2.044 & 2.115 \\
$\mu_{1}$ & -0.713 & -0.188 & -0.011 & 0.135 & 0.372 \\
$\mu_{2}$ & -3.228 & -1.845 & -1.205 & -0.223 & 0.198 \\
$\mu_{3}$ & -6.107 & -2.501 & -1.710 & -1.057 & 2.107 \\
$\mu_{4}$ & -7.927 & -3.132 & -1.704 & -0.359 & 4.011 \\
$\phi_{1}$ & -0.418 & -0.061 & 0.149 & 0.381 & 0.919 \\
$\phi_{2}$ & -0.476 & 0.055 & 0.332 & 0.668 & 1.447 \\
$\phi_{3}$ & -1.987 & -0.086 & 0.417 & 0.959 & 2.912 \\
$\phi_{4}$ & -2.975 & -0.418 & 0.420 & 1.283 & 3.899 \\
$a_{1}$ & 0.416 & 0.724 & 0.860 & 0.988 & 1.246 \\
$a_{2}$ & 0.760 & 1.526 & 1.741 & 1.880 & 2.145 \\
$a_{3}$ & -1.925 & 1.098 & 1.851 & 2.505 & 5.309 \\
$a_{4}$ & -3.503 & 0.536 & 1.800 & 3.011 & 6.818 \\
\hline
\end{tabular}

Table 10: Posterior quantile estimates 


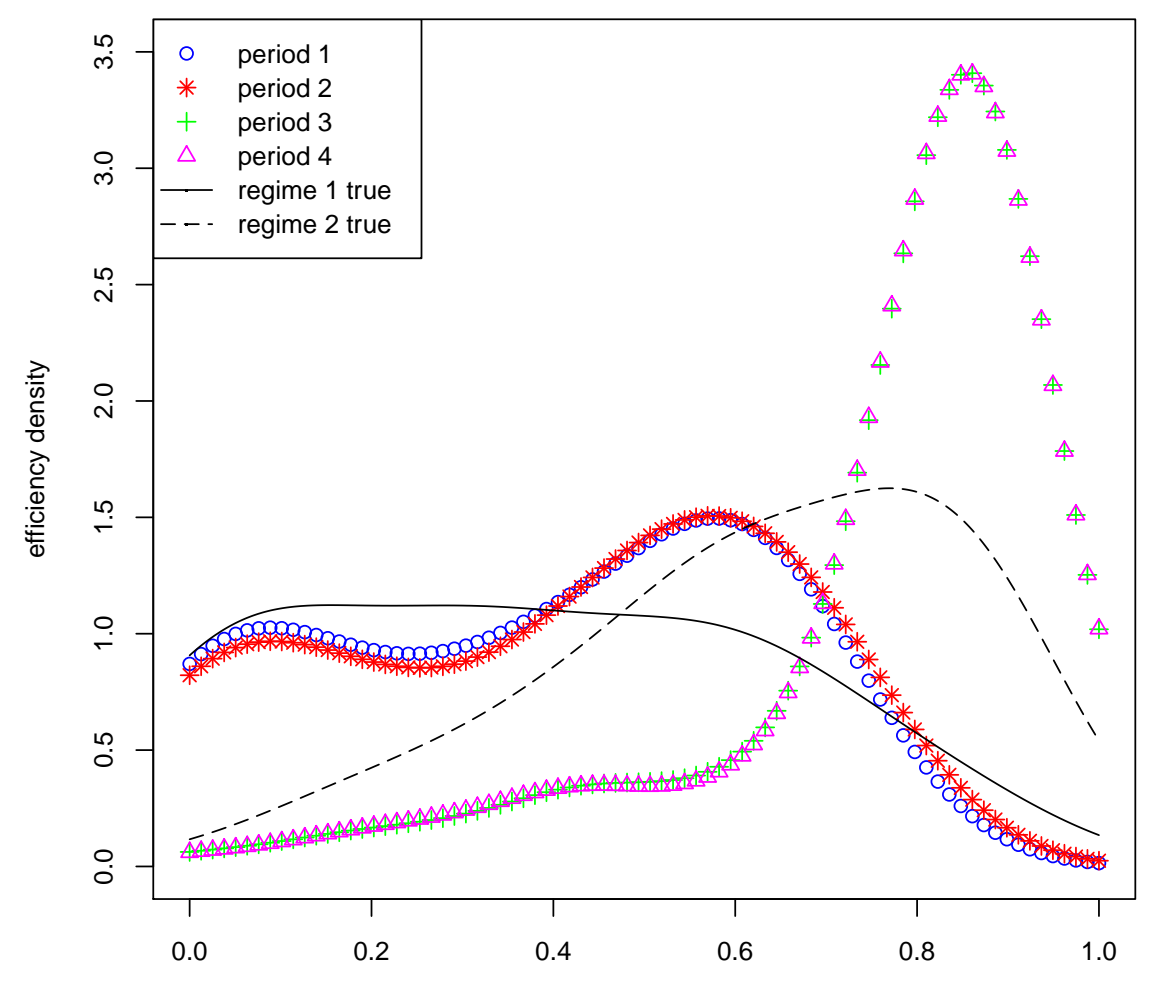

Figure 2: Plots of the efficiency densities for artificial data 
average our variables every 3 years to make $T=16$. In order to apply our method developed in previous sections, one more thing is worth mentioning. Our basic model assumes that all the individuals have the same production technology (i.e. the same slopes and intercept) for a given period. For our dataset, this may not be valid. It should be sensible to separate the economic agents into different groups with different technologies. Here we separate our data into two samples: one comprised by the richest countries or regions, and the other the poorest based on the real per capita income (rgdpch) in 2007. Each sample consists of $N=36$ countries or regions. The rich group is composed of those with real per capita income in the top one third quantile while the poor group are those in the bottom one third quantile. We then estimate our econometric model using these two samples separately. Note that 27 out of 36 rich countries or regions remain rich in our sample period and 25 out of 36 stay poor in the poor group. It is therefore reasonable to assume that these two groups have different technology parameters.

We assume all the slopes and intercept can change over time (i.e. $\beta=0$ ). The empirical results are obtained after 20,000 draws with the initial 2,000 draws discarded. For both groups, we can see that the efficiency seems to have less regimes than the technology and the locations of the breakpoints tend to be different. Judging from Figure 3, the efficiency breakpoints of the rich group are likely to happen in the early 1960s and 1980s. The red square line represents the prior. As for the number of regimes, the data confirm and reinforce the number to be 1 to 2 since at these two points, the posterior probabilities are higher than the prior. With regard to the breakpoint locations, the first point in the graph indicates the probability with which the breakpoint does not exist. The data suggest that the first breakpoint may not exist. But if it exists, it is most likely to happen in the early 1960s, the end of 1970s or early 1980s. Figure 4 presents the estimation results for efficiency of the rich group along with the probabilities of period $t$ being a breakpoint. Note that the estimation results are obainted by averaging different models, which are defined by different number of regimes and different locations of breakpoints. Estimates of $\mu$ and $\phi$, which are defined in (2), gradually decrease over time. This implies a slight improvement and a tighter distribution of efficiency. The bottom right corner of Figure 4 shows the density plots of the efficiency medians of different countries or regions in the year of 1960,1981 and $2005^{8}$. It is not hard to notice that the distribution tends to get tighter to the middle over time. The means and standard deviations of the medians for these three years are respectively: $62.92 \%$ and $12.63 \%, 63.96 \%$ and $11.39 \%, 64.36 \%$ and $8.7 \%$. To sum up, the efficiency for the rich group as a whole seems to have been improved slightly over the years. For the poor group, the efficiency results are quite different. Figure 5 shows that there is not much evidence supporting any structrual changes. The data suggest there should be only one regime and no breakpoints exist. Figure 4 confirms these results. The values of $\mu$ and $\phi$ are almost flat over time and the density plots of the medians

\footnotetext{
${ }^{8}$ Note that each of this data point is the average of three years. For example, 1960 is the average of 1960, 1961 and 1962 .
} 


\begin{tabular}{|c|c|c|c|c|c|c|c|c|}
\hline rank & country & efficiency & rank & country & efficiency & rank & country & efficiency \\
\hline 1 & United States & 0.84 & 13 & Australia & 0.70 & 25 & Barbados & 0.59 \\
\hline 2 & Luxembourg & 0.84 & 14 & Argentina & 0.70 & 26 & Chile & 0.59 \\
\hline 3 & Canada & 0.79 & 15 & Austria & 0.68 & 27 & Greece & 0.59 \\
\hline 4 & New Zealand & 0.77 & 16 & Norway & 0.68 & 28 & Finland & 0.54 \\
\hline 5 & Netherlands & 0.76 & 17 & France & 0.67 & 29 & Hong Kong & 0.50 \\
\hline 6 & Puerto Rico & 0.75 & 18 & Iceland & 0.65 & 30 & Japan & 0.50 \\
\hline 7 & Equatorial Guinea & 0.74 & 19 & Mauritius & 0.65 & 31 & Portugal & 0.48 \\
\hline 8 & United Kingdom & 0.74 & 20 & Israel & 0.64 & 32 & Malaysia & 0.46 \\
\hline 9 & Switzerland & 0.73 & 21 & Italy & 0.61 & 33 & Taiwan & 0.43 \\
\hline 10 & Sweden & 0.71 & 22 & Trinidad Tobago & 0.61 & 34 & Singapore & 0.41 \\
\hline 11 & Denmark & 0.70 & 23 & Ireland & 0.59 & 35 & Korea,Rep. & 0.38 \\
\hline 12 & Belgium & 0.70 & 24 & Spain & 0.59 & 36 & Cyprus & 0.35 \\
\hline
\end{tabular}

Table 11: Efficiency ranking for the rich group in 1960

\begin{tabular}{|c|c|c|c|c|c|c|c|c|}
\hline rank & country & efficiency & rank & country & efficiency & rank & country & efficiency \\
\hline 1 & Puerto Rico & 0.81 & 13 & Argentina & 0.67 & 25 & Taiwan & 0.61 \\
\hline 2 & Luxembourg & 0.80 & 14 & Denmark & 0.66 & 26 & Chile & 0.61 \\
\hline 3 & United States & 0.78 & 15 & Italy & 0.66 & 27 & Finland & 0.60 \\
\hline 4 & United Kingdom & 0.74 & 16 & Australia & 0.66 & 28 & Spain & 0.60 \\
\hline 5 & Mauritius & 0.73 & 17 & Hong Kong & 0.66 & 29 & Barbados & 0.59 \\
\hline 6 & Netherlands & 0.72 & 18 & Switzerland & 0.65 & 30 & Trinidad Tobago & 0.58 \\
\hline 7 & Sweden & 0.72 & 19 & New Zealand & 0.65 & 31 & Japan & 0.56 \\
\hline 8 & Norway & 0.72 & 20 & Greece & 0.64 & 32 & Malaysia & 0.54 \\
\hline 9 & Canada & 0.71 & 21 & Ireland & 0.63 & 33 & Singapore & 0.52 \\
\hline 10 & France & 0.70 & 22 & Israel & 0.63 & 34 & Cyprus & 0.51 \\
\hline 11 & Belgium & 0.70 & 23 & Iceland & 0.61 & 35 & Portugal & 0.50 \\
\hline 12 & Austria & 0.69 & 24 & Equatorial Guinea & 0.61 & 36 & Korea,Rep. & 0.41 \\
\hline
\end{tabular}

Table 12: Efficiency ranking for the rich group in 2005

of different years overlap each other. The means of the medians range from $31.37 \%$ to $31.42 \%$ and the standard deviation is about $10 \%$. Although for both rich and poor group as a whole, we do not see much change in efficiency, it is still worth investigating the individual efficiencies and the rankings. Table 11 and Table 12 show the efficiency medians of the rich group in 1960 and 2005 respectively. We can see that the rankings are not the same for the two tables. Table 13 shows the efficiency change between 1960 and 2005. Note that the top six efficiency gainers, namely, Taiwan, Cyprus, Hong Kong, Singapore, Mauritius and Malaysia, did not used to be rich in 1960. Part of their economic success should be due to improvement in production efficiency. The related results for the poor group are shown in Table 14, Table 15 and Table 16. It is easy to notice that the rankings and the levels of efficiencies for the poor group are almost identical for the two periods selected. The members of the poor group have not made much progress in terms of efficiency over the years.

We now turn to the analysis of production technology. There seem to be more 


\section{number of regimes}

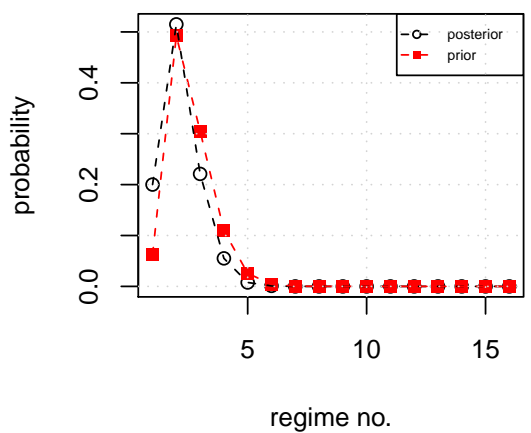

breakpoint 2

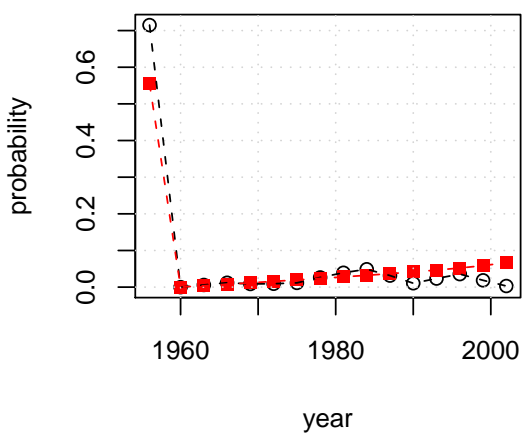

breakpoint 1

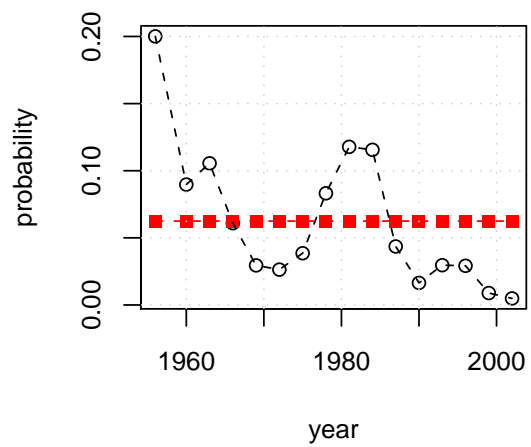

breakpoint 3

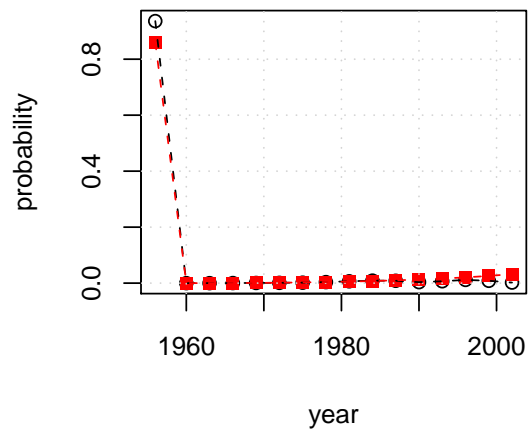

Figure 3: Probabilities for number of efficiency regimes and breakpoint locations for the rich group

\begin{tabular}{|r|l|r|r|l|r|r|l|l|}
\hline rank & country & change & rank & country & change & rank & country & change \\
\hline 1 & Taiwan & 0.18 & 13 & Ireland & 0.04 & 25 & Argentina \\
2 & Cyprus & 0.16 & 14 & France & 0.03 & 26 & Trinidad Tobago \\
3 & Hong Kong & 0.15 & 15 & Korea,Rep. & 0.02 & 27 & Iceland \\
4 & Singapore & 0.11 & 16 & Portugal & 0.02 & 28 & Luxembourg \\
5 & Mauritius & 0.09 & 17 & Chile & 0.02 & 29 & Denmark \\
6 & Malaysia & 0.07 & 18 & Sweden & 0.01 & 30 & Netherlands \\
7 & Puerto Rico & 0.07 & 19 & Austria & 0.01 & 31 & Australia \\
8 & Finland & 0.06 & 20 & Spain & 0.01 & 32 & United States \\
9 & Japan & 0.06 & 21 & Barbados & 0.00 & 33 & Switzerland \\
10 & Greece & 0.05 & 22 & Belgium & 0.00 & 34 & Canada \\
11 & Italy & 0.05 & 23 & United Kingdom & 0.00 & 35 & New Zealand \\
12 & Norway & 0.04 & 24 & Israel & -0.01 & -0.04 \\
\end{tabular}

Table 13: Efficiency changes for the rich group between 1960 and 2005 

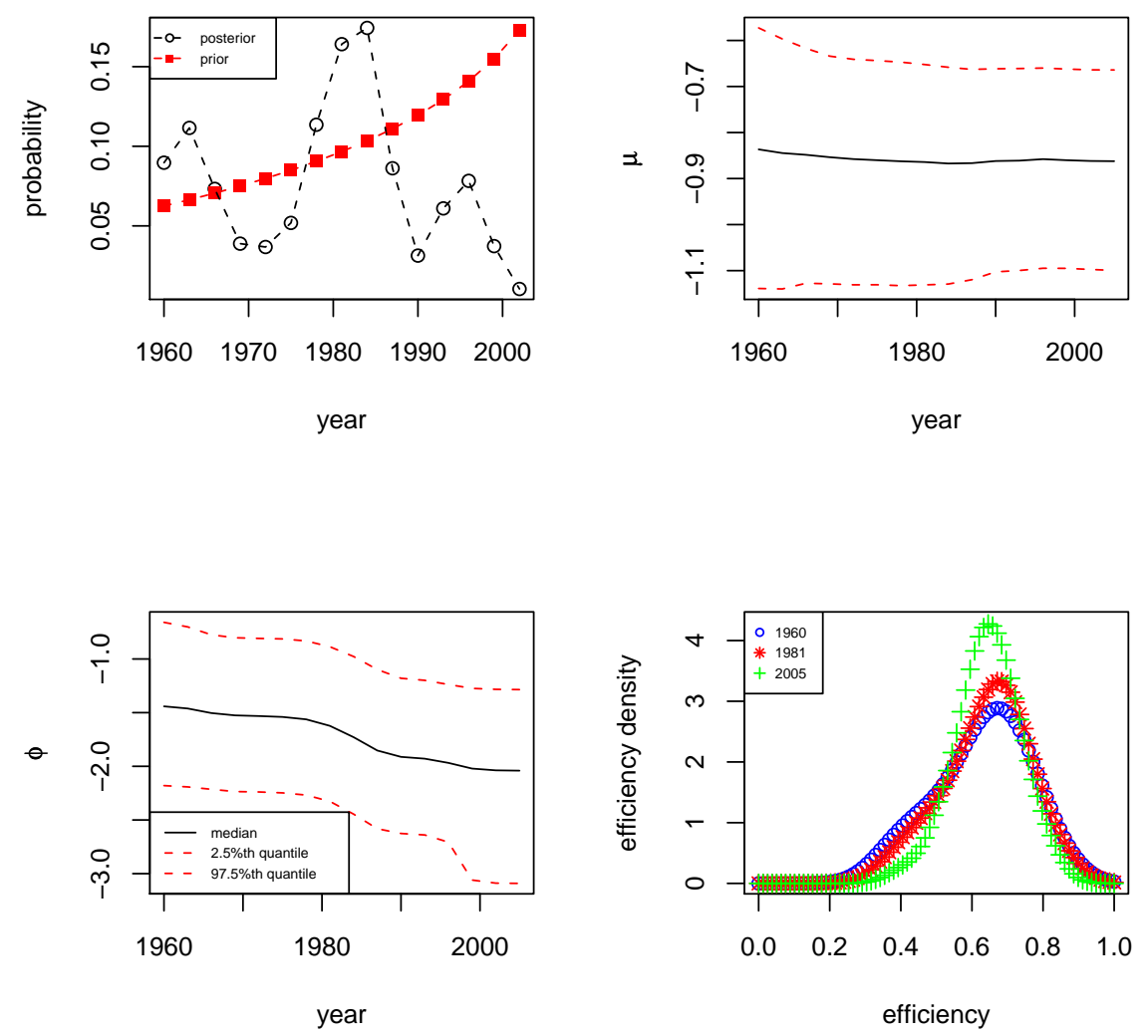

Figure 4: Probabilities of period t being a breakpoint and efficiency estimates for the rich group 

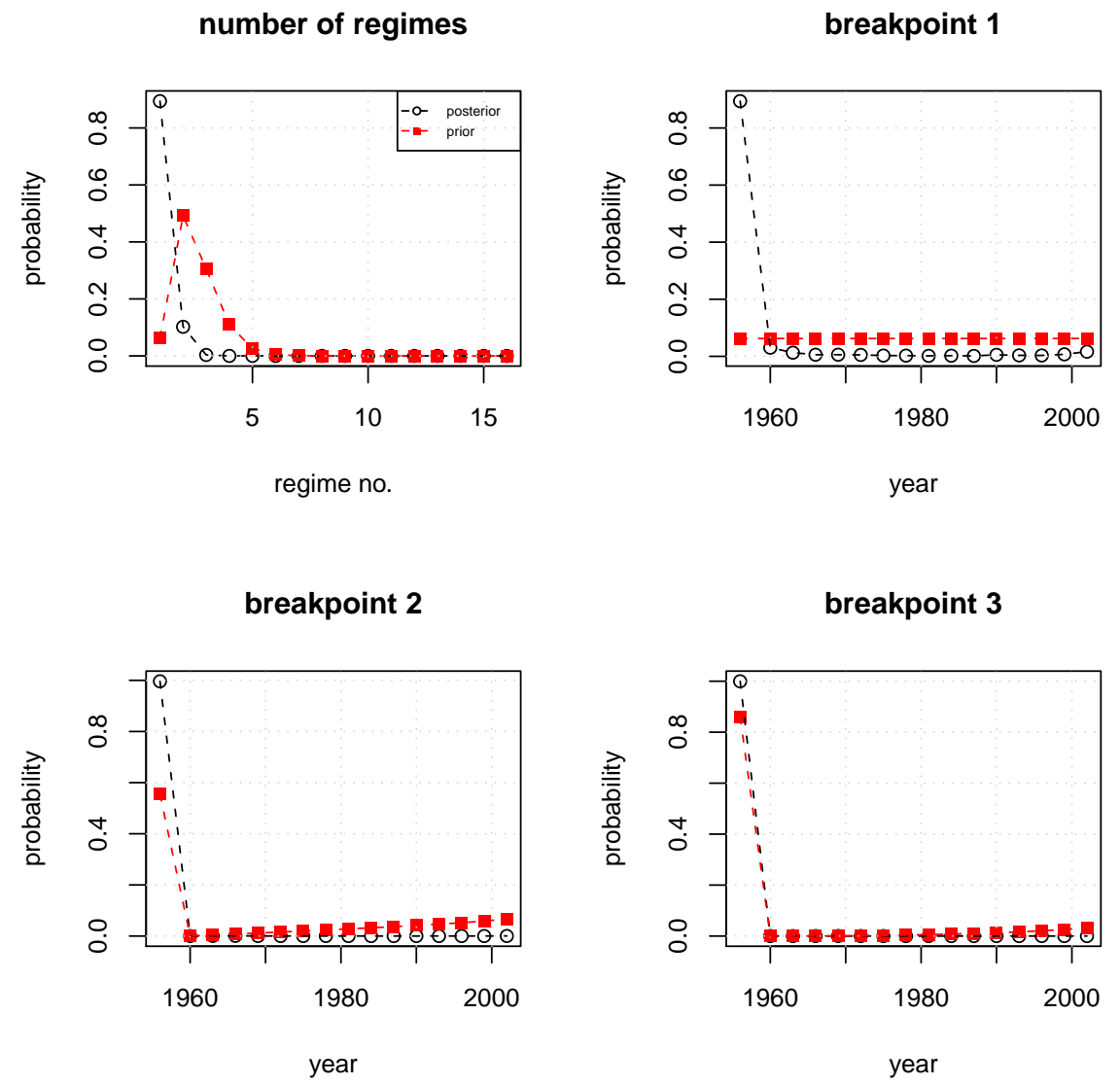

Figure 5: Probabilities for number of efficiency regimes and breakpoint locations for the poor group 

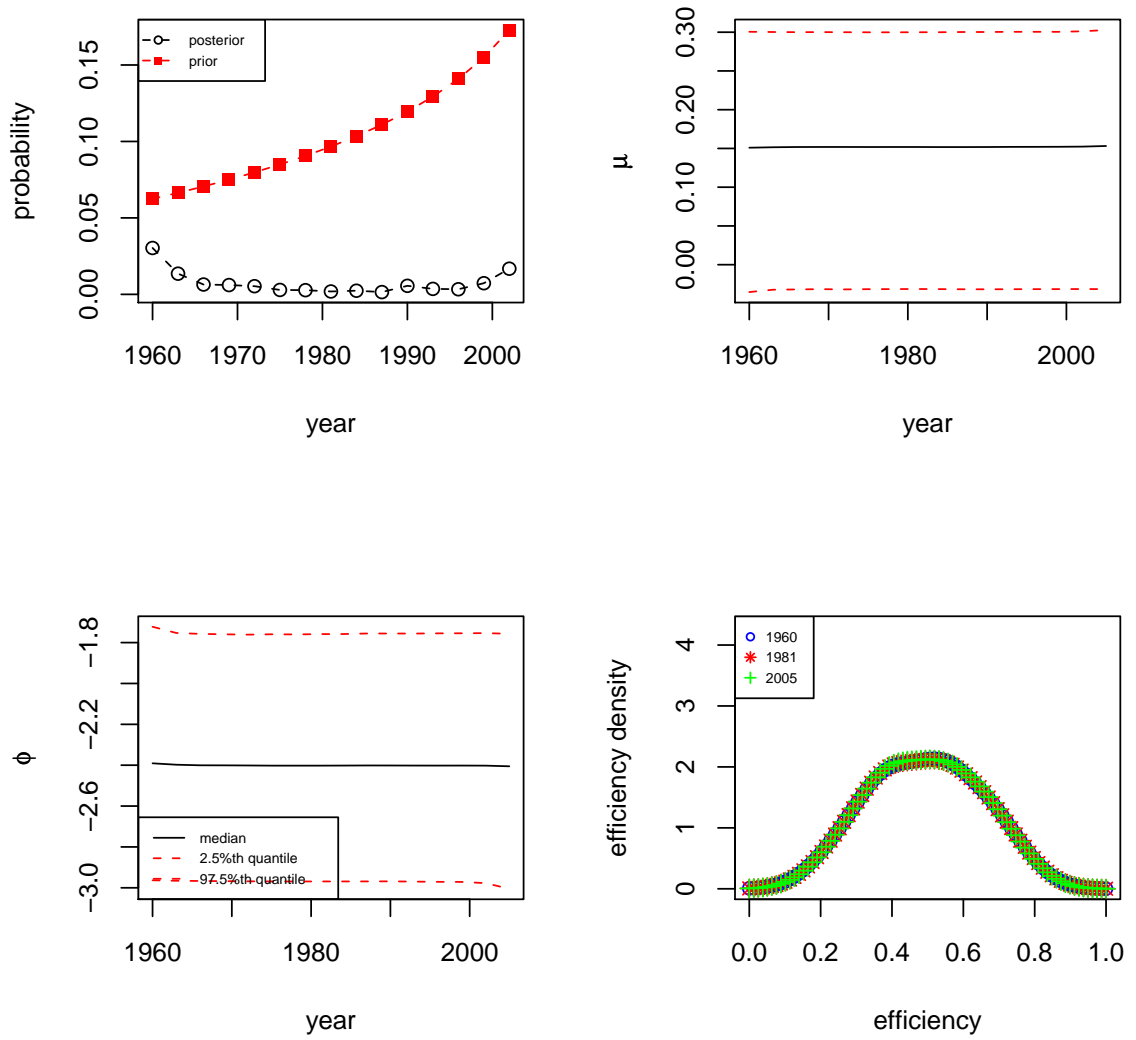

Figure 6: Probabilities of period t being a breakpoint and efficiency estimates for the poor group 


\begin{tabular}{|c|c|c|c|c|c|c|c|c|}
\hline rank & country & efficiency & rank & country & efficiency & rank & country & efficiency \\
\hline 1 & Congo,Rep. & 0.52 & 13 & Haiti & 0.33 & 25 & Ethiopia & 0.27 \\
\hline 2 & Zimbabwe & 0.51 & 14 & Bangladesh & 0.33 & 26 & Papua New Guinea & 0.26 \\
\hline 3 & Cote d'Ivoire & 0.49 & 15 & Congo,Dem.Rep. & 0.33 & 27 & Benin & 0.25 \\
\hline 4 & Syria & 0.48 & 16 & Rwanda & 0.32 & 28 & Cent.African Rep. & 0.25 \\
\hline 5 & Cameroon & 0.47 & 17 & Uganda & 0.31 & 29 & Ghana & 0.24 \\
\hline 6 & Senegal & 0.46 & 18 & Zambia & 0.30 & 30 & Burundi & 0.24 \\
\hline 7 & Chad & 0.42 & 19 & Gambia & 0.29 & 31 & Togo & 0.22 \\
\hline 8 & Nigeria & 0.38 & 20 & Mali & 0.29 & 32 & Burkina Faso & 0.21 \\
\hline 9 & Nicaragua & 0.36 & 21 & Niger & 0.28 & 33 & Lesotho & 0.20 \\
\hline 10 & Mozambique & 0.35 & 22 & Madagascar & 0.28 & 34 & Malawi & 0.18 \\
\hline 11 & Kenya & 0.35 & 23 & Mauritania & 0.28 & 35 & Tanzania & 0.16 \\
\hline 12 & Comoros & 0.34 & 24 & Nepal & 0.27 & 36 & Guinea-Bissau & 0.12 \\
\hline
\end{tabular}

Table 14: Efficiency ranking for the poor group in 1960

\begin{tabular}{|r|l|r|r|l|r|r|l|r|}
\hline rank & country & efficiency & rank & country & efficiency & rank & country & efficiency \\
\hline 1 & Congo,Rep. & 0.53 & 13 & Haiti & 0.33 & 25 & Ethiopia & 0.27 \\
2 & Zimbabwe & 0.51 & 14 & Bangladesh & 0.32 & 26 & Papua New Guinea & 0.26 \\
3 & Cote d'Ivoire & 0.50 & 15 & Congo,Dem.Rep. & 0.32 & 27 & Benin & 0.26 \\
4 & Syria & 0.48 & 16 & Rwanda & 0.31 & 28 & Cent.African Rep. & 0.25 \\
5 & Cameroon & 0.47 & 17 & Uganda & 0.31 & 29 & Ghana & 0.25 \\
6 & Senegal & 0.45 & 18 & Zambia & 0.29 & 30 & Burundi & 0.24 \\
7 & Chad & 0.41 & 19 & Mali & 0.29 & 31 & Togo & 0.22 \\
8 & Nigeria & 0.39 & 20 & Gambia & 0.28 & 32 & Burkina Faso & 0.21 \\
9 & Mozambique & 0.35 & 21 & Mauritania & 0.28 & 33 & Lesotho & 0.20 \\
10 & Nicaragua & 0.35 & 22 & Niger & 0.28 & 34 & Malawi & 0.18 \\
11 & Comoros & 0.34 & 23 & Madagascar & 0.28 & 35 & Tanzania & 0.16 \\
12 & Kenya & 0.34 & 24 & Nepal & 0.27 & 36 & Guinea-Bissau & 0.12 \\
\hline
\end{tabular}

Table 15: Efficiency ranking for the poor group in 2005

\begin{tabular}{|r|l|r|r|l|r|r|l|r|}
\hline rank & \multicolumn{1}{|c|}{ country } & change & rank & \multicolumn{1}{|c|}{ country } & change & rank & country & change \\
\hline 1 & Congo,Rep. & 0.01 & 13 & Ethiopia & 0 & 25 & Kenya & 0.00 \\
2 & Ghana & 0.01 & 14 & Tanzania & 0 & 26 & Nepal & 0.00 \\
3 & Cote d'Ivoire & 0.01 & 15 & Cameroon & 0 & 27 & Rwanda & 0.00 \\
4 & Mauritania & 0.00 & 16 & Zimbabwe & 0 & 28 & Haiti & 0.00 \\
5 & Comoros & 0.00 & 17 & Madagascar & 0 & 29 & Bangladesh & 0.00 \\
6 & Syria & 0.00 & 18 & Cent.African Rep. & 0 & 30 & Niger & 0.00 \\
7 & Nigeria & 0.00 & 19 & Togo & 0 & 31 & Zambia & -0.01 \\
8 & Malawi & 0.00 & 20 & Burundi & 0 & 32 & Gambia & -0.01 \\
9 & Papua New Guinea & 0.00 & 21 & Burkina Faso & 0 & 33 & Chad & -0.01 \\
10 & Benin & 0.00 & 22 & Mozambique & 0 & 34 & Nicaragua & -0.01 \\
11 & Guinea-Bissau & 0.00 & 23 & Uganda & 0 & 35 & Senegal & -0.01 \\
12 & Mali & 0.00 & 24 & Lesotho & 0 & 36 & Congo,Dem.Rep. & -0.01 \\
\hline
\end{tabular}

Table 16: Efficiency changes for the poor group between 1960 and 2005 


\begin{tabular}{rlrrlrrrr}
\hline \hline rank & \multicolumn{1}{c}{ country } & efficiency & rank & country & efficiency & rank & country & efficiency \\
\hline 1 & United States & 0.81 & 10 & Denmark & 0.66 & 19 & Ireland \\
2 & Luxembourg & 0.75 & 11 & Belgium & 0.65 & 20 & Iceland & 0.58 \\
3 & Canada & 0.74 & 12 & Switzerland & 0.65 & 21 & Spain & 0.57 \\
4 & United Kingdom & 0.73 & 13 & France & 0.65 & 22 & Greece & 0.57 \\
5 & Puerto Rico & 0.72 & 14 & Australia & 0.64 & 23 & Barbados & 0.57 \\
6 & New Zealand & 0.70 & 15 & Austria & 0.64 & 24 & Finland & 0.52 \\
7 & Netherlands & 0.70 & 16 & Norway & 0.62 & 25 & Japan \\
8 & Argentina & 0.69 & 17 & Italy & 0.59 & & 0.50 \\
9 & Sweden & 0.68 & 18 & Israel & 0.59 & & \\
\hline
\end{tabular}

Table 17: Efficiency ranking for the rich group in 1960

patterns for both groups than efficiency. For the rich group, the data support 5 to 6 regimes with breakpoints at almost every decade. The breakpoint pattern seems simpler for the poor group. The data support at most two regimes with the breakpoint most likely to happen at the end of 1960s and in the 1970s. For both groups, there seems to be a trend showing that the capital elasticity increases over time while the labour elasticity tends to decrease. For the poor group, such trend seems to have stopped after 1980 while there are more ups and downs for the rich group over the sample period studied. The rich group appears to have experienced more regime changes. For the intercept, it keeps rising until 1975 and then gradually comes down before the abrupt fall in the early 1990s. It then rises again in the middle 1990s. The labour elasticity has similar patterns apart from the small fall in the early 1960s. As for the capital elasticity, it always goes to the opposite direction to that of labour whenever there is a regime shift. One thing to note is that for a given period, the sum of labour and capital elasticity is approximately 1 , which implies constant returns to scale.

To check the robustness of our results, we estimate two more samples. One sample consists of the countries that have been in the top one third quantile (the richest 36 countries) every year from 1960 to 2007, while the other sample are those that appear in the bottom third quantile throughout the sample period. There are 25 countries in the rich group and 17 in the poor group. Those countries that join and leave the rich and poor groups in the sample period are left out. We just keep the countries that have been consistent for the two samples. By doing so, for example, the four Asian Tigers along with some other countries are not included in the rich group sample. The results are more or less similar to what we previously found. A main difference for the rich group is that we now have weaker evidence for two efficiency regimes. If there is a breakpoint in the sample period, it is likely to occur only in the early 1960s. We can not see another breakpoint in the early 1980s. For the poor group, as before there is not much evidence of regime changes in efficiency. Since these are the 19 countries that have been consistently poor, averagely speaking, their eifficiency levels are lower than the poorest 36 countries in 2007 we studied previously.

In terms of the production technology regimes and breakpoints, for the 25 
number of regimes

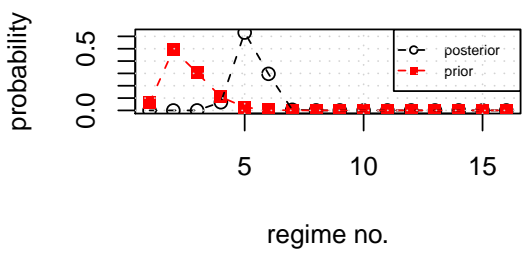

breakpoint 2

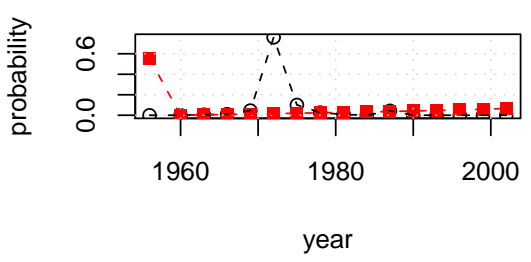

breakpoint 4

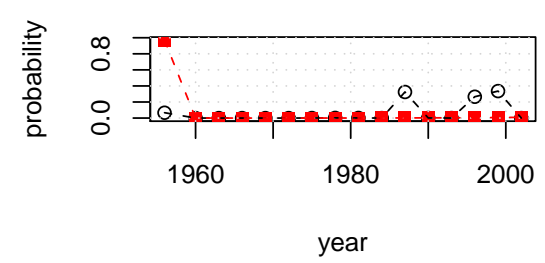

breakpoint 1

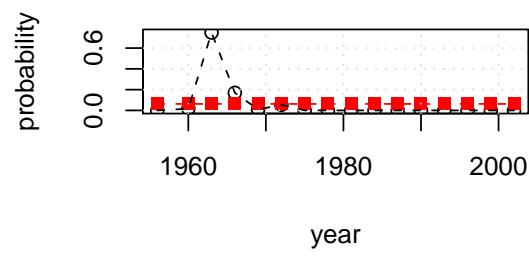

breakpoint 3

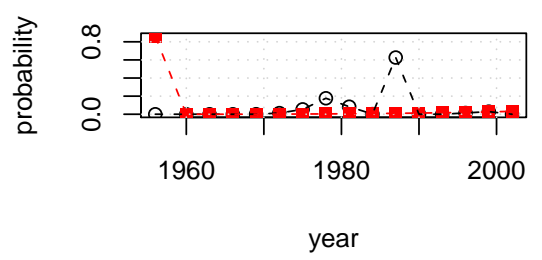

breakpoint 5

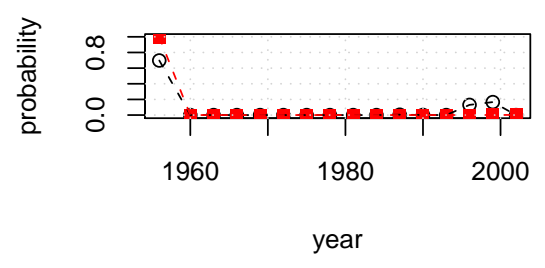

Figure 7: Probabilities for number of technology regimes and breakpoint locations for the rich group

\begin{tabular}{rlrrlrrlr}
\hline \hline rank & country & efficiency & rank & country & efficiency & rank & country & efficiency \\
\hline 1 & United States & 0.79 & 10 & Belgium & 0.67 & 19 & Israel & 0.60 \\
2 & Puerto Rico & 0.78 & 11 & New Zealand & 0.66 & 20 & Spain & 0.58 \\
3 & Luxembourg & 0.76 & 12 & Austria & 0.65 & 21 & Ireland & 0.58 \\
4 & United Kingdom & 0.74 & 13 & Denmark & 0.65 & 22 & Iceland & 0.57 \\
5 & Canada & 0.71 & 14 & Norway & 0.65 & 23 & Barbados & 0.55 \\
6 & Netherlands & 0.71 & 15 & Switzerland & 0.64 & 24 & Finland & 0.54 \\
7 & Sweden & 0.69 & 16 & Australia & 0.64 & 25 & Japan & 0.53 \\
8 & Argentina & 0.68 & 17 & Italy & 0.63 & & & \\
9 & France & 0.67 & 18 & Greece & 0.60 & & & \\
\hline
\end{tabular}

Table 18: Efficiency ranking for the rich group in 2005 

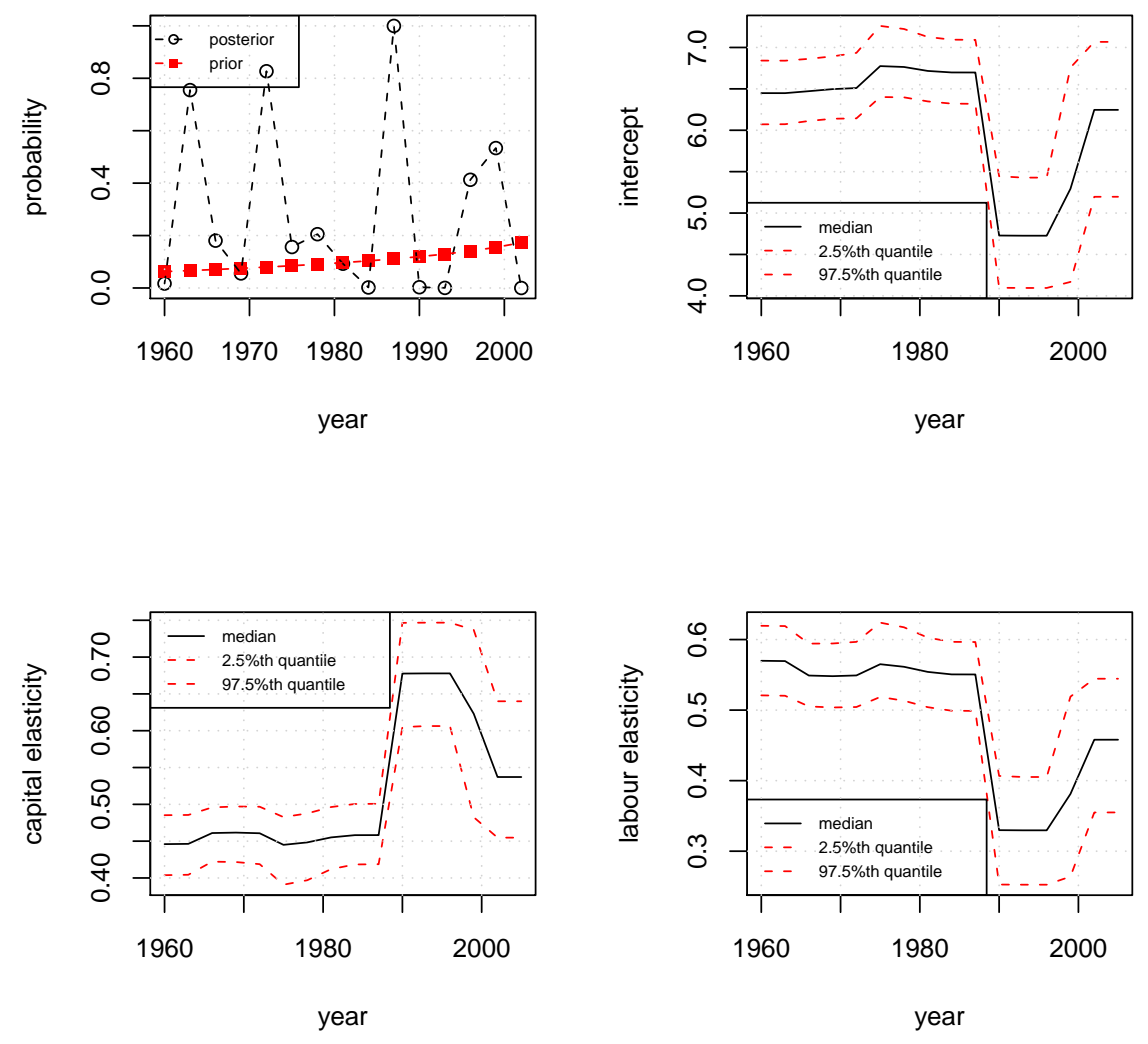

Figure 8: Probabilities of period t being a breakpoint and technology parameter estimates for the rich group

\begin{tabular}{rlrrlrrrr}
\hline \hline rank & country & efficiency & rank & country & efficiency & rank & country & efficiency \\
\hline 1 & Puerto Rico & 0.05 & 10 & Spain & 0.01 & 19 & Iceland & -0.01 \\
2 & Italy & 0.04 & 11 & Israel & 0.01 & 20 & Argentina & -0.01 \\
3 & Greece & 0.03 & 12 & Barbados & 0.01 & 21 & Switzerland & -0.01 \\
4 & Japan & 0.03 & 13 & Sweden & 0.01 & 22 & Denmark & -0.01 \\
5 & France & 0.03 & 14 & Luxembourg & 0.01 & 23 & United States & -0.02 \\
6 & Finland & 0.03 & 15 & Netherlands & 0.01 & 24 & Canada & -0.03 \\
7 & Norway & 0.02 & 16 & Ireland & 0.00 & 25 & New Zealand & -0.04 \\
8 & Austria & 0.02 & 17 & United Kingdom & 0.00 & & & \\
9 & Belgium & 0.02 & 18 & Australia & -0.01 & & & \\
\hline
\end{tabular}

Table 19: Efficiency changes for the rich group between 1960 and 2005 


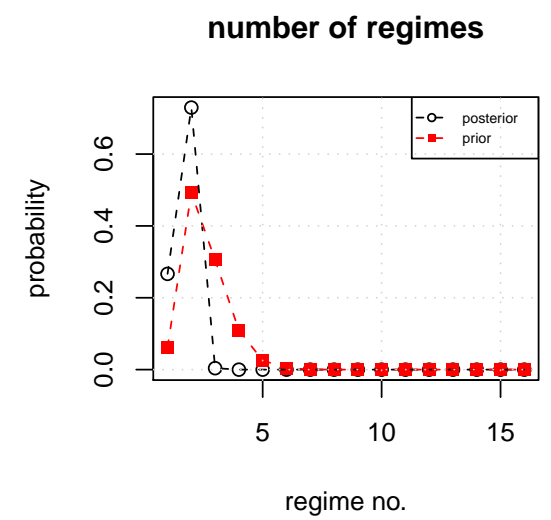

breakpoint 2

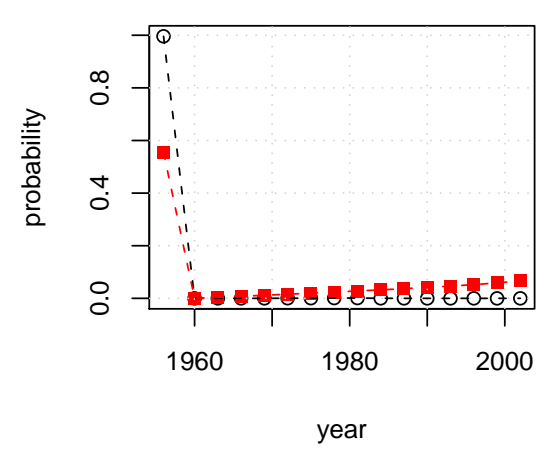

breakpoint 1

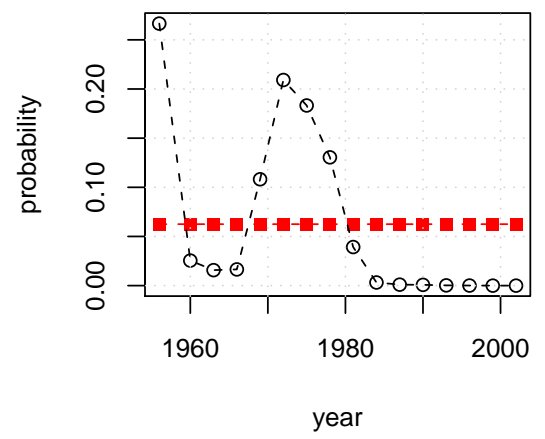

breakpoint 3

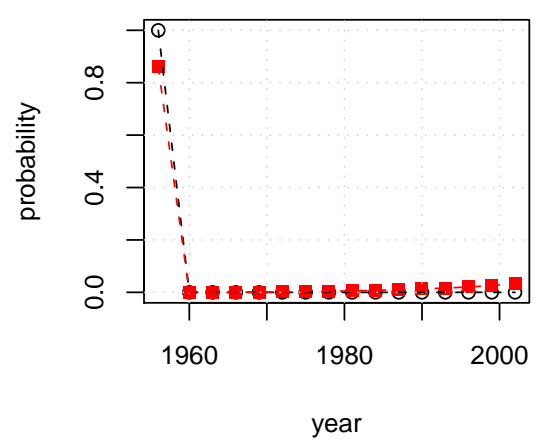

Figure 9: Probabilities for number of technology regimes and breakpoint locations for the poor group

\begin{tabular}{rlrrlr}
\hline \hline rank & country & efficiency & rank & \multicolumn{1}{c}{ country } & efficiency \\
\hline 1 & Mozambique & 0.23 & 11 & Cent.African Rep. & 0.18 \\
2 & Niger & 0.22 & 12 & Ghana & 0.18 \\
3 & Nepal & 0.21 & 13 & Togo & 0.18 \\
4 & Gambia & 0.21 & 14 & Lesotho & 0.16 \\
5 & Rwanda & 0.21 & 15 & Burkina Faso & 0.15 \\
6 & Mali & 0.20 & 16 & Burundi & 0.15 \\
7 & Uganda & 0.19 & 17 & Malawi & 0.13 \\
8 & Benin & 0.19 & 18 & Tanzania & 0.11 \\
9 & Madagascar & 0.19 & 19 & Guinea-Bissau & 0.09 \\
10 & Ethiopia & 0.19 & & & \\
\hline
\end{tabular}

Table 20: Efficiency ranking for the poor group in 1960 


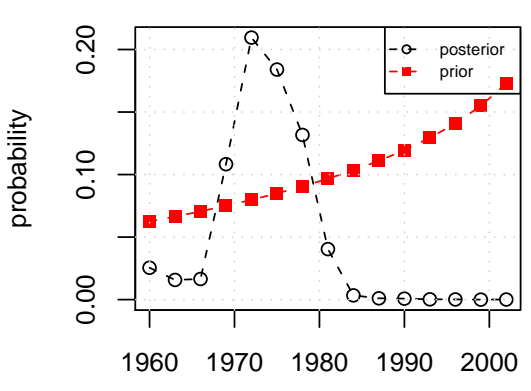

year

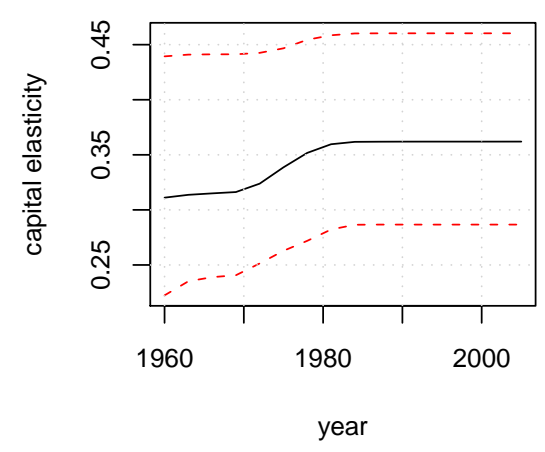

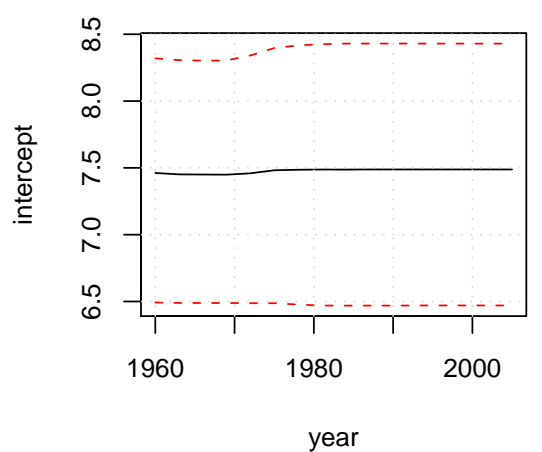

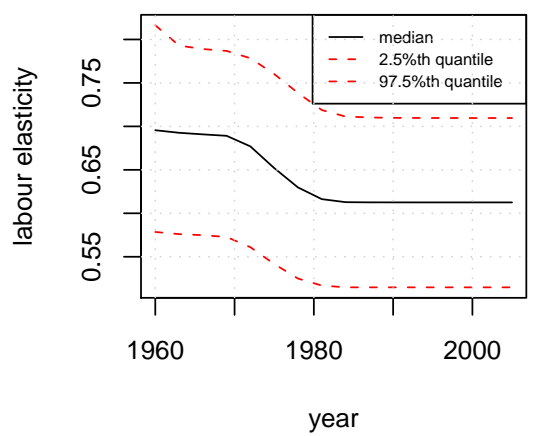

Figure 10: Probabilities of period t being a breakpoint and technology parameter estimates for the poor group

\begin{tabular}{rlrrlr}
\hline \hline rank & country & efficiency & rank & \multicolumn{1}{c}{ country } & efficiency \\
\hline 1 & Mozambique & 0.23 & 11 & Ethiopia & 0.18 \\
2 & Mali & 0.21 & 12 & Cent.African Rep. & 0.18 \\
3 & Nepal & 0.21 & 13 & Togo & 0.17 \\
4 & Niger & 0.20 & 14 & Lesotho & 0.16 \\
5 & Rwanda & 0.20 & 15 & Burkina Faso & 0.15 \\
6 & Gambia & 0.20 & 16 & Burundi & 0.15 \\
7 & Benin & 0.20 & 17 & Malawi & 0.14 \\
8 & Ghana & 0.20 & 18 & Tanzania & 0.11 \\
9 & Uganda & 0.19 & 19 & Guinea-Bissau & 0.10 \\
10 & Madagascar & 0.19 & & & \\
\hline
\end{tabular}

Table 21: Efficiency ranking for the poor group in 2005 


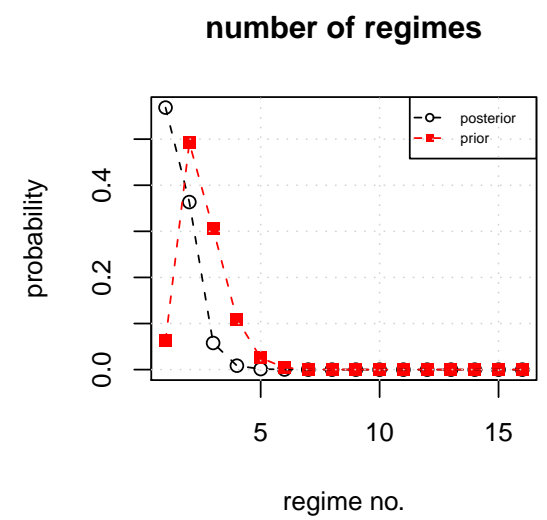

breakpoint 2

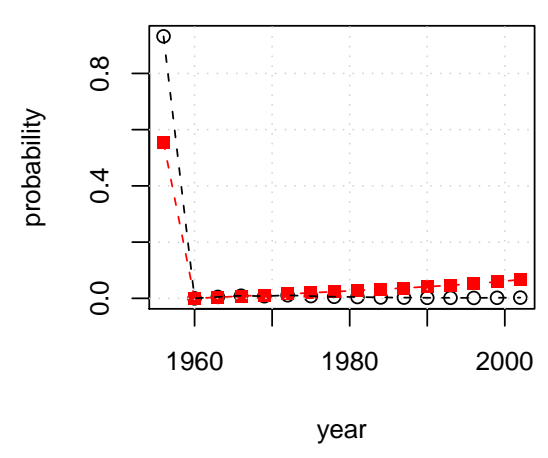

breakpoint 1

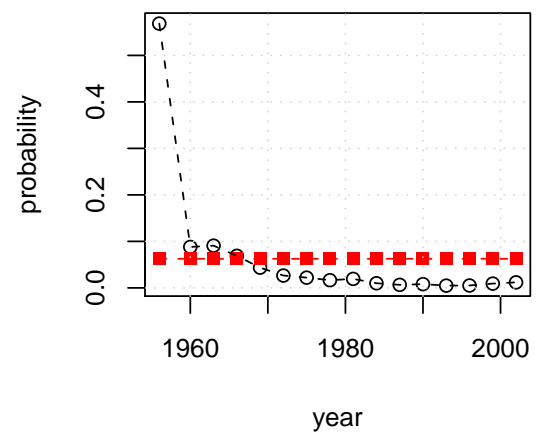

breakpoint 3

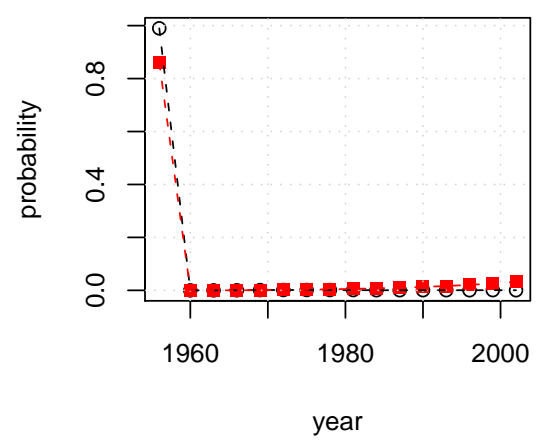

Figure 11: Probabilities for number of efficiency regimes and breakpoint locations for the rich group of 25 countries

\begin{tabular}{rlrrlr}
\hline \hline rank & country & efficiency & rank & \multicolumn{1}{c}{ country } & efficiency \\
\hline 1 & Malawi & 0.02 & 11 & Madagascar & 0.00 \\
2 & Ghana & 0.02 & 12 & Uganda & 0.00 \\
3 & Mali & 0.01 & 13 & Ethiopia & 0.00 \\
4 & Guinea-Bissau & 0.01 & 14 & Togo & 0.00 \\
5 & Benin & 0.01 & 15 & Cent.African Rep. & 0.00 \\
6 & Lesotho & 0.01 & 16 & Nepal & -0.01 \\
7 & Burundi & 0.00 & 17 & Rwanda & -0.01 \\
8 & Tanzania & 0.00 & 18 & Gambia & -0.01 \\
9 & Burkina Faso & 0.00 & 19 & Niger & -0.02 \\
10 & Mozambique & 0.00 & & & \\
\hline
\end{tabular}

Table 22: Efficiency changes for the poor group between 1960 and 2005 

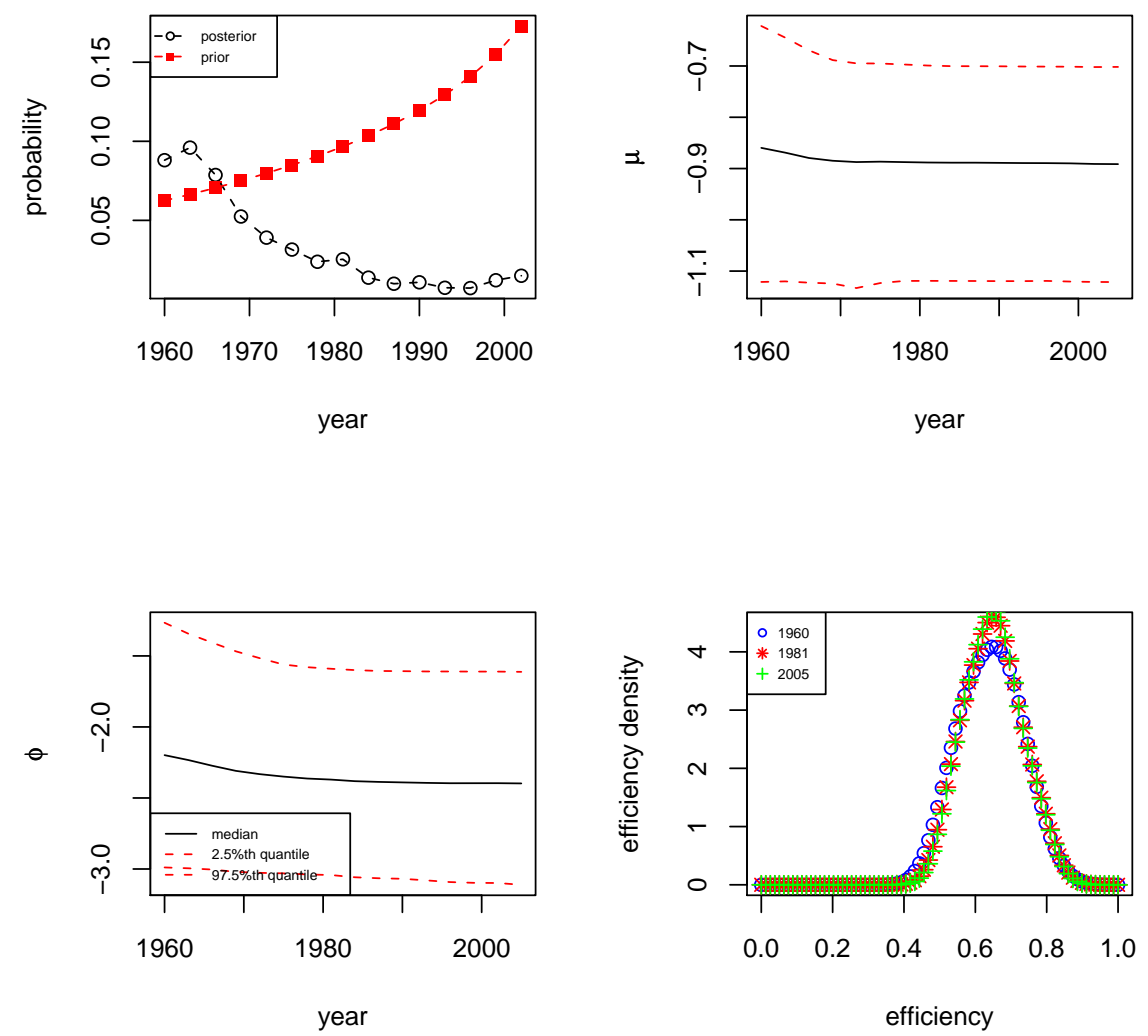

Figure 12: Probabilities of period t being a breakpoint and efficiency estimates for the rich group of 25 countries 

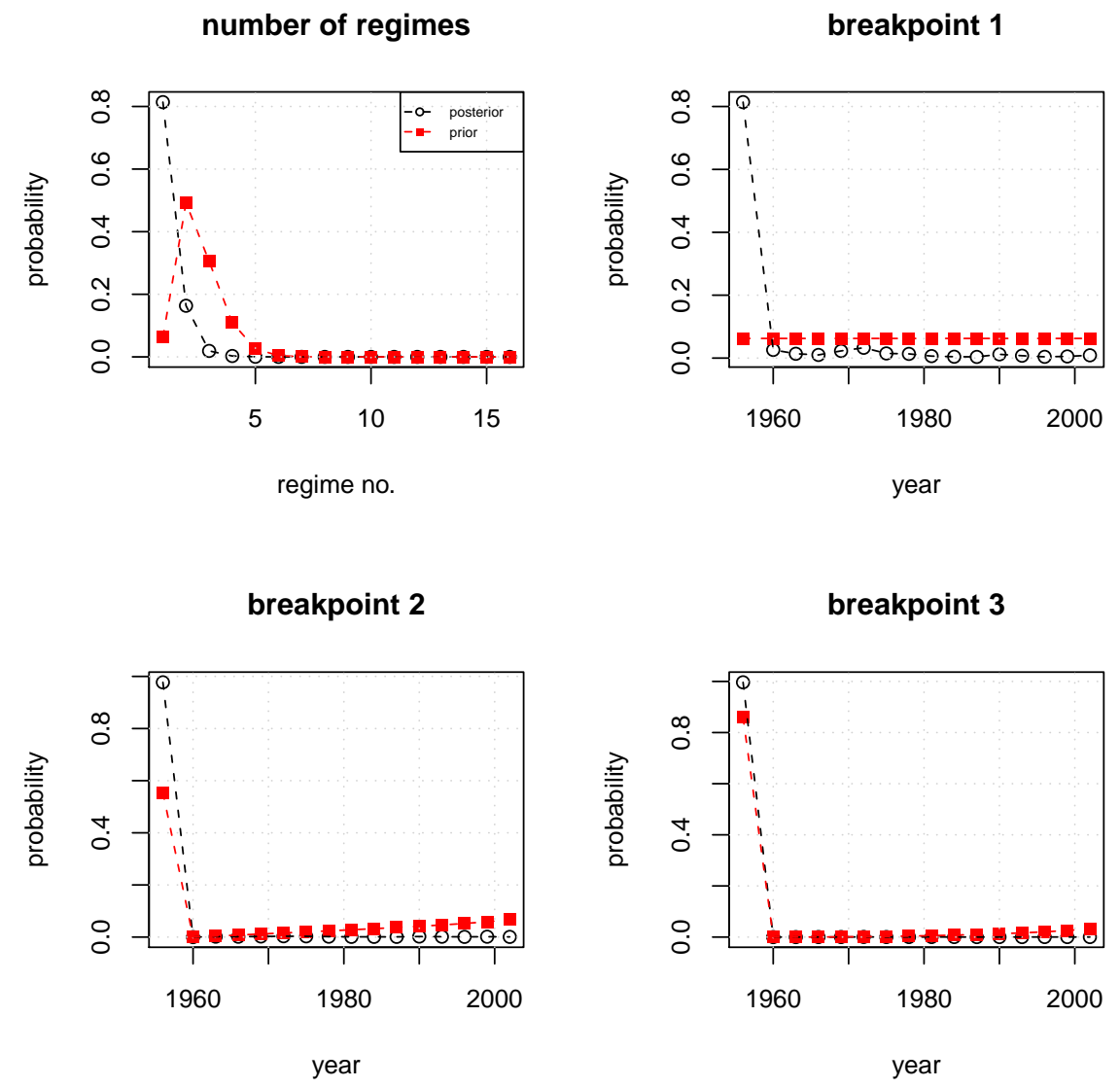

Figure 13: Probabilities for number of efficiency regimes and breakpoint locations for the poor group of 19 countries 

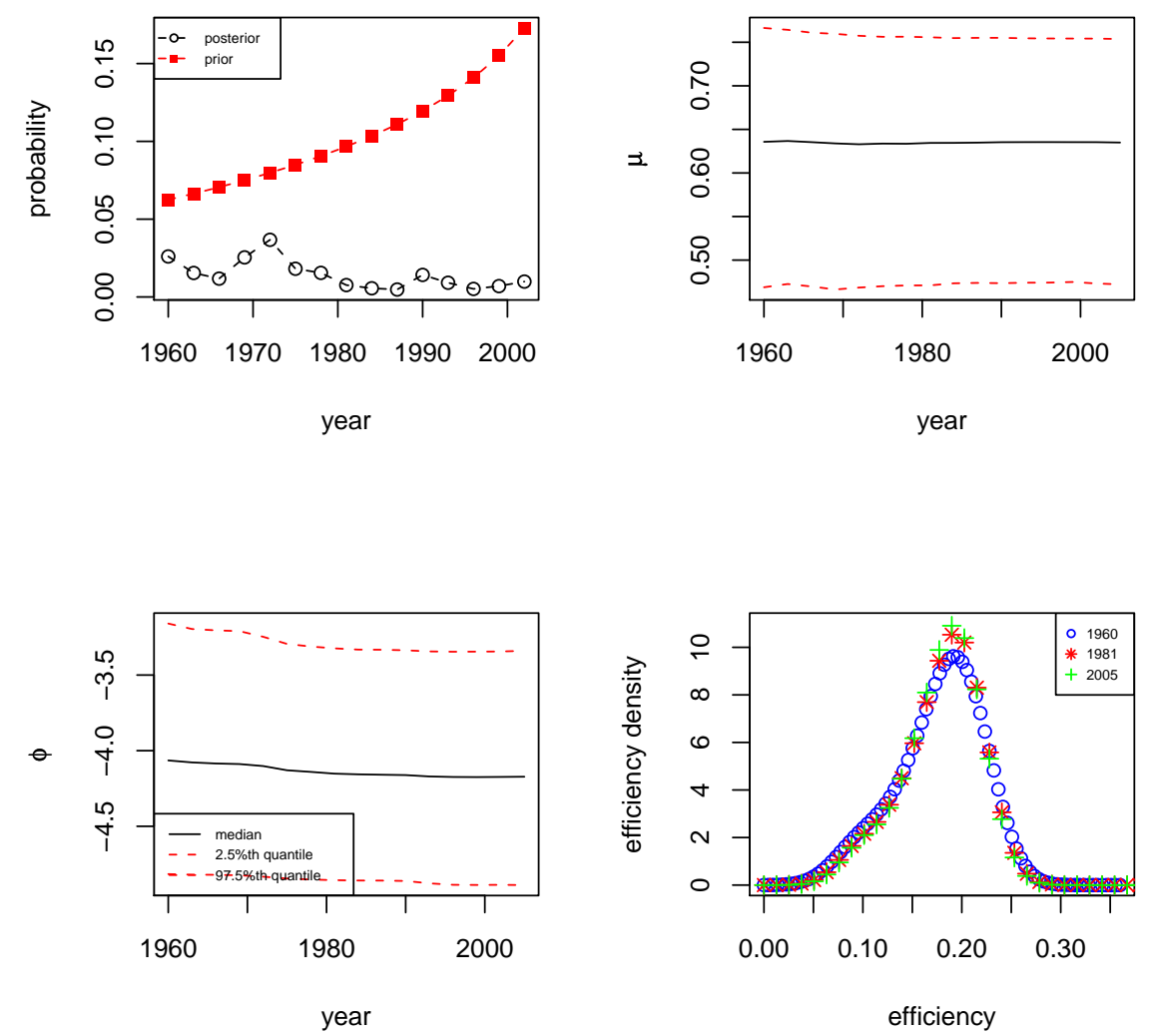

Figure 14: Probabilities of period t being a breakpoint and efficiency estimates for the poor group of 19 countires 
rich countries, there is one less regime than the previous case of 36 countries. The breakpoints are present for every decade except for the 1990s. While the intercept and the labour elasticity have somewhat inverted U-shapes, the capital elasticity has a notable rise in the early 1990s and remain relatively constant since then. This finding is different from the case of 36 countries where there is a fall in capital elasticity and a rise in labour elasticity and intercept in the middle of 1990s. For the poor countries, compared to the 36 countries before, there seem to be one more regime and one more breakpoint, which occurs in the early 1990s. Both the intercept and the labour elasticity trend downward over the years, while the capital elasticity goes up.

number of regimes

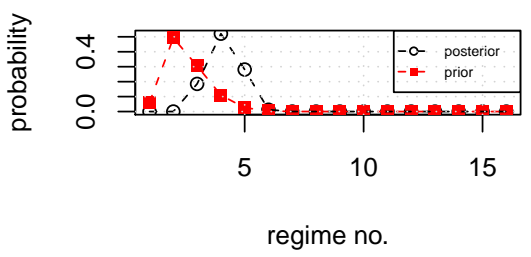

breakpoint 2

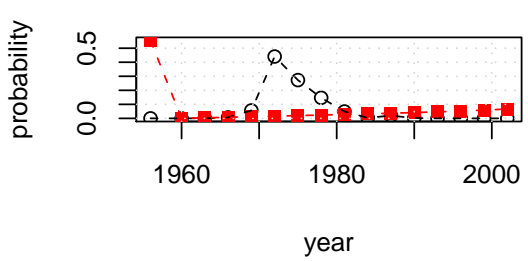

breakpoint 4

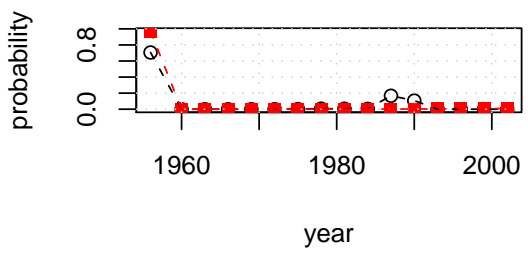

breakpoint 1

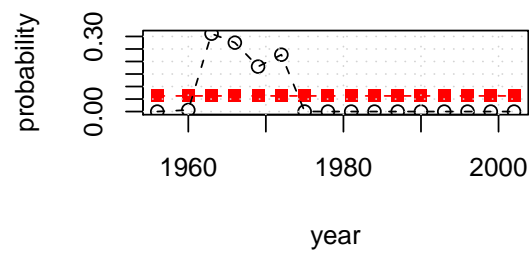

breakpoint 3

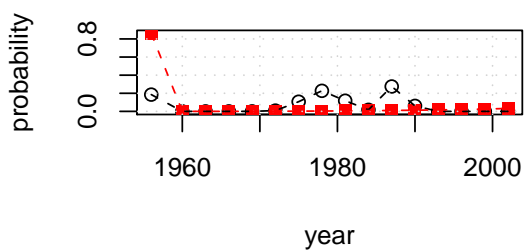

breakpoint 5

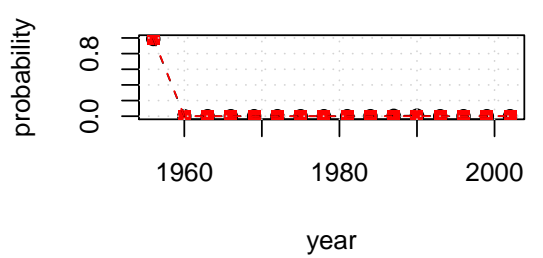

Figure 15: Probabilities for number of technology regimes and breakpoint locations for the rich group of 25 countries

Note that the sum of capital and labour elasticity is approximately one for both the rich and the poor group, which suggests constant returns to scale. 

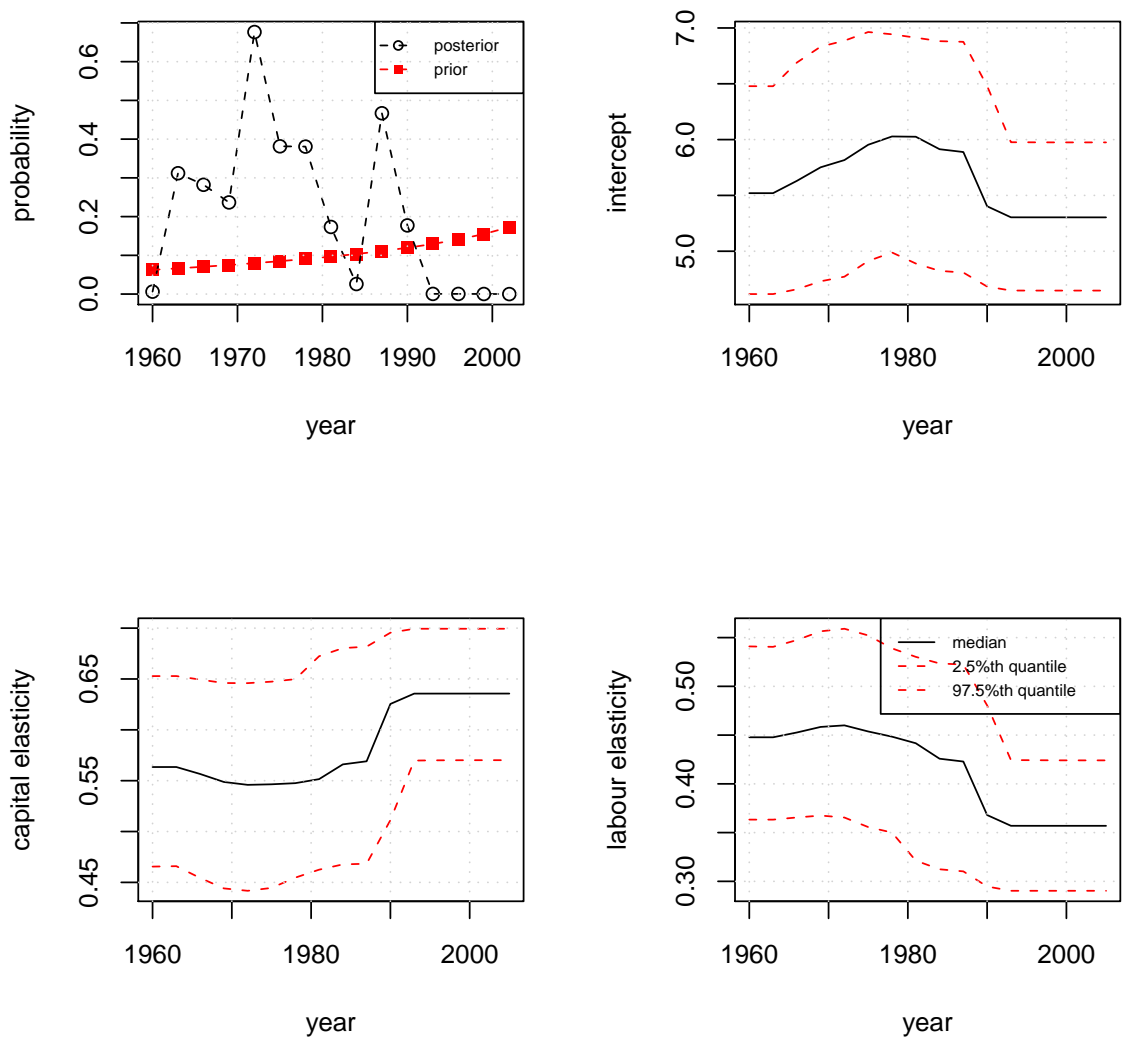

Figure 16: Probabilities of period $t$ being a breakpoint and technology parameter estimates for the rich group of 25 countries 

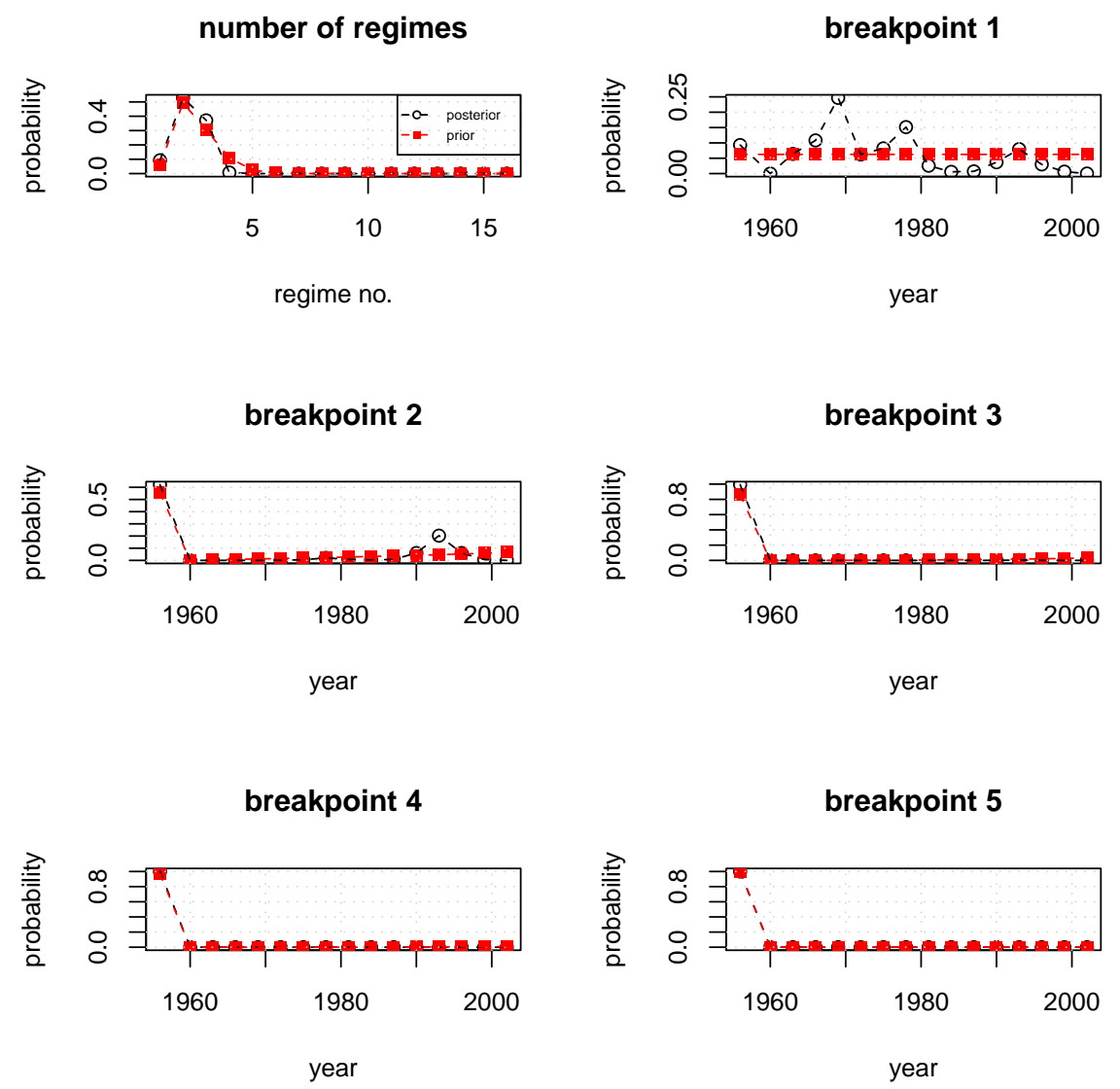

Figure 17: Probabilities for number of technology regimes and breakpoint locations for the poor group of 19 countries 

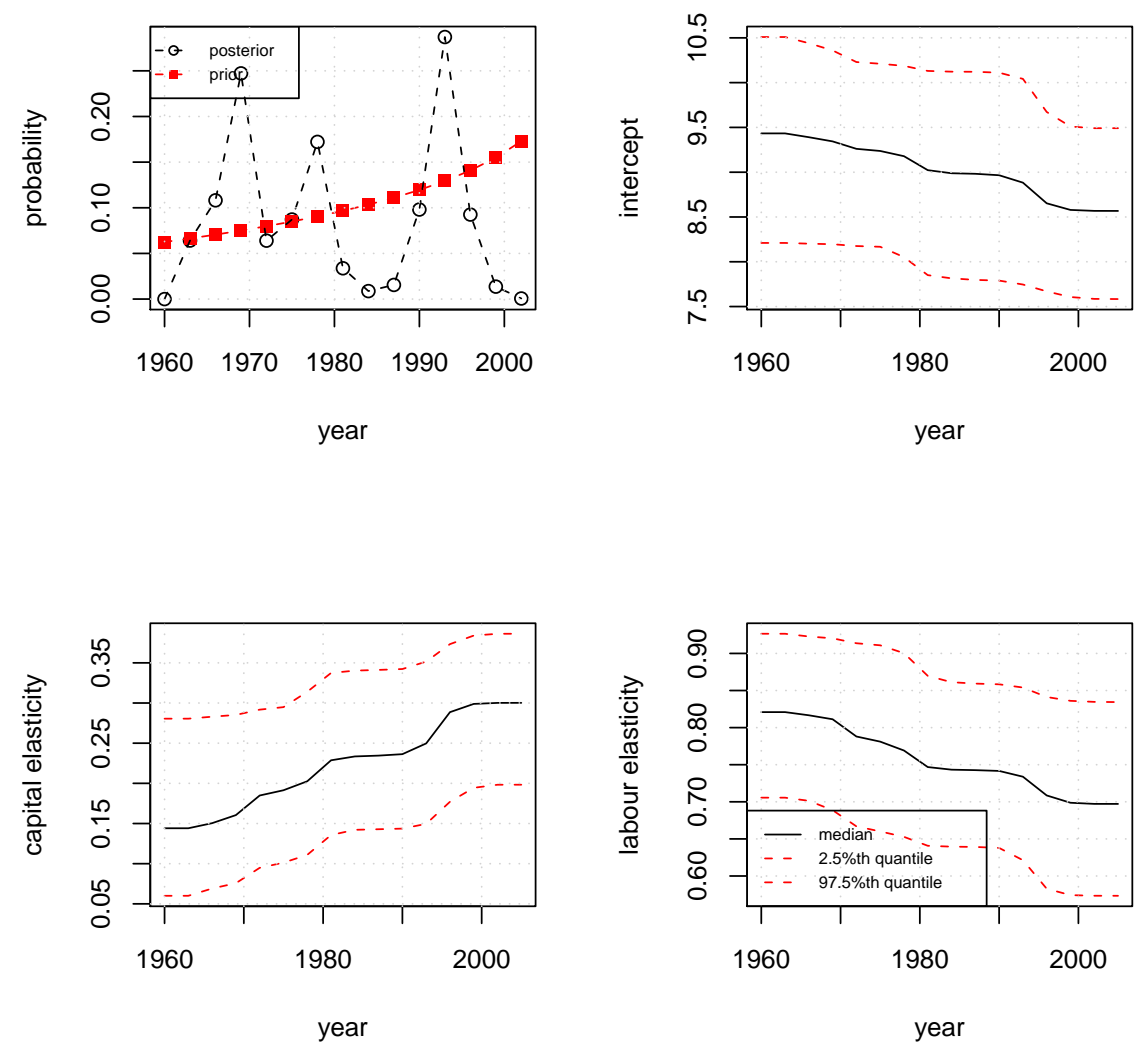

Figure 18: Probabilities of period $t$ being a breakpoint and technology parameter estimates for the poor group of 19 countries 
Next we impose this restriction and rewrite our model as,

$$
y-x_{L}=a+\left(x_{K}-x_{L}\right) a_{K}-\exp (\gamma)+\epsilon .
$$

For notational simplicity, we drop all the it subscripts here. Equation (13) states that the frontier of the natural $\log$ of per worker output $\left(y-x_{L}\right)$ is a linear function of the log of per worker capital. Figure 19 and 20 show the estimated frontiers with the log of per worker output and capital substituted by their cross-sectional sample average for the rich and the poor group for every period and the intercept and capital elasticity evaluated at their posterior medians. The actual per worker output and capital are also shown in the graph. We can see that though there are ups and downs for both the rich and poor countries, the frontier and the actual per worker output improve gradually over the years for the rich, while they remain at about the same level for the poor. We can also see that there are gaps between the frontier and the actual values for both the rich and the poor. For the rich, the ratio of the actual over the frontier is around $0.6-0.69$, while it is around $0.07-0.1$ for the poor, which means there is bigger room of improvement for the poor than the rich given their respective available technology.

\section{Conclusion}

In this paper, we have proposed a stochastic frontier model with unknown number of structural breaks and unknown locations of break points in both efficiency and technology. The structural changes in efficiency and technology are allowed to happen separately, while the changes in efficiency involve both conditional

means and variances. Exercises with artificial data show that the strength of panel data can help to identify these two kinds of changes even if the panel is short. The implementation difficulties with panel data are discussed. Relevant computing techniques and estimation strategies are proposed to overcome such obstacles. Our methods are applied to study the world production. We find that rich countries or regions have experienced a few regime changes in both production technology and production efficiency over the period of 1960 to 2007, while the poor countries and regions have remained relatively stagnant. The efficiency for the poor group appears to be almost identical in the sample period. For the world as a whole, the recent labour elasticity seems to have fallen while the capital elasticity has increased compared to 1960 . We have not found many structural changes in efficiency for our empirical application. For other applications in future research, where many efficiency changes are expected, it may be beneficial to incorporate factors that could explain such changes into our framework.

\section{References}

Aigner, D., K. Lovell, And P. Schmidt (1977): "Formulation and estimation of stochastic frontier production function models," Journal of Economet- 


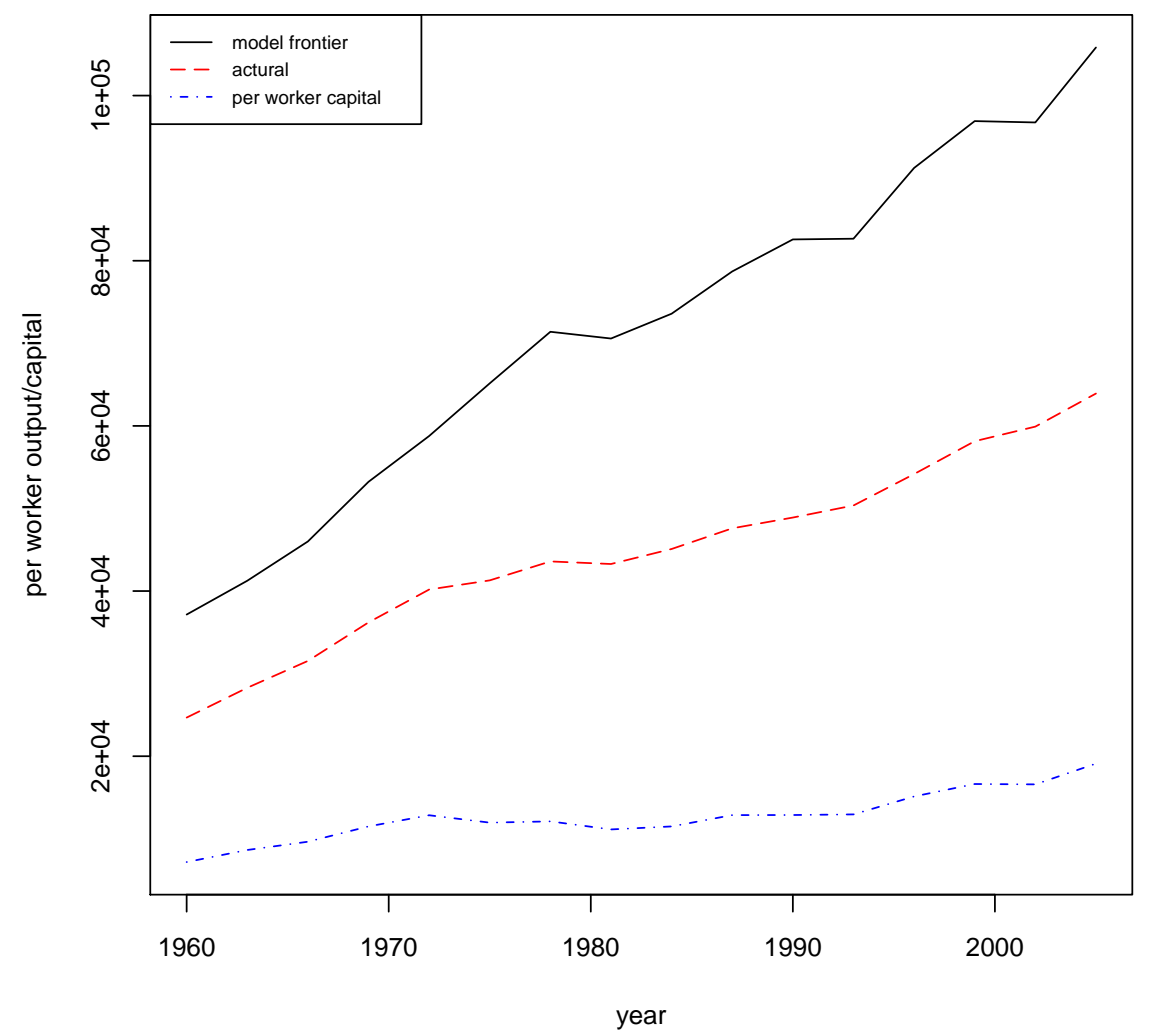

Figure 19: The estimated per worker output frontier for the rich group of 25 countries 


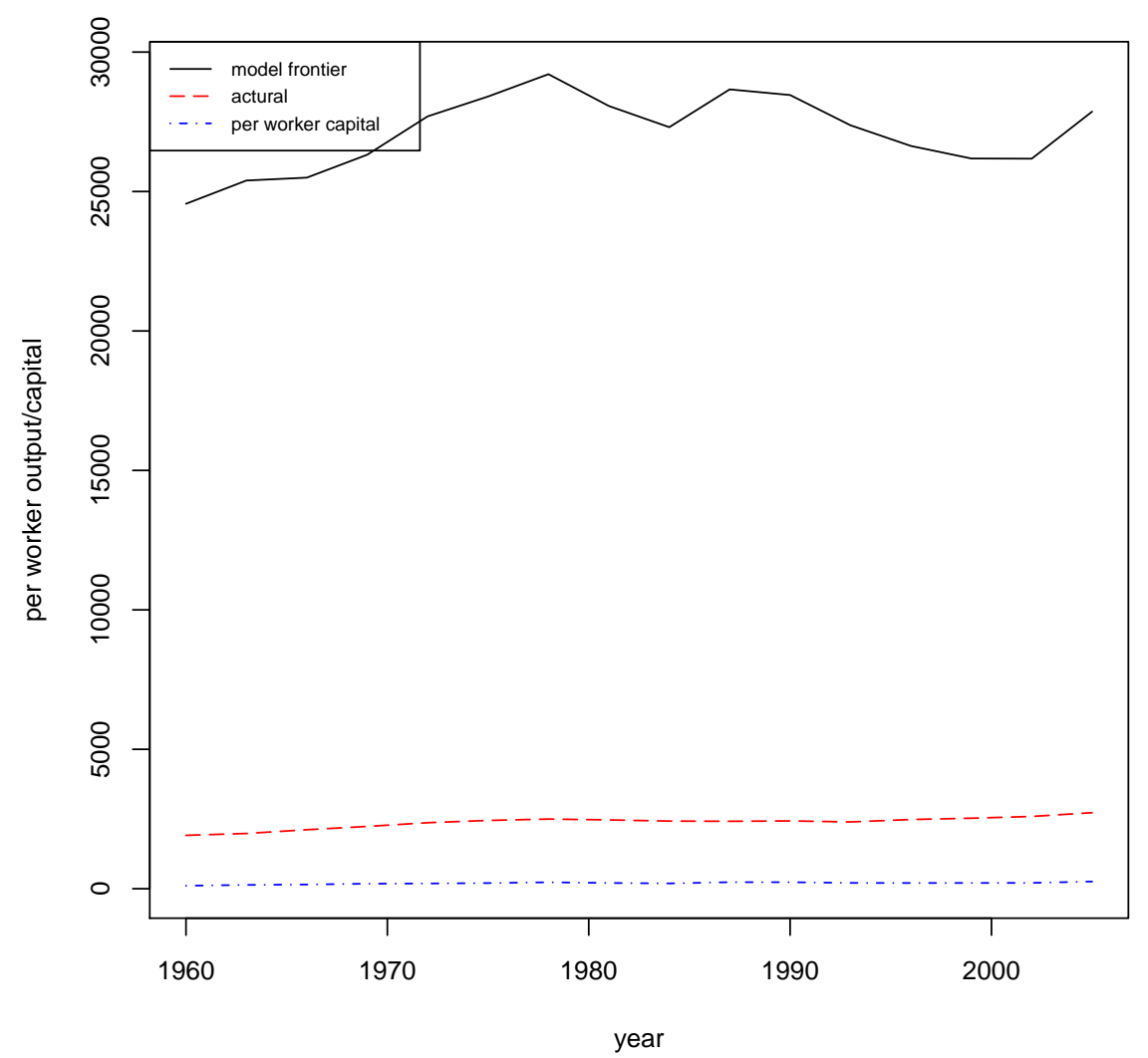

Figure 20: The estimated per worker output frontier for the poor group of 19 countries 
rics, 6, 21-37.

Carter, C. And R. Kohn (1994): "On Gibbs Sampling for State Space Models," Biometrika, 81, 541-553.

CHIB, S. (1998): "Estimation and Comparison of Multiple Change-Point Models," Journal of Econometrics, 86, 221-241.

De Jong, P. And N. Shephard (1995): "The Simulation Smoother for Time Series Models," Biometrika, 82, 339-350.

Fernandez, C., E. Ley, And M. F. Steel (2001): "Benchmark Priors for Bayesian Model Averaging," Journal of Econometrics, 100, 381-427.

Fraley, C. And A. Raftery (2009): mclust: Model-Based Clustering / Normal Mixture Modeling, r package version 3.3.1.

Gerlach, R., C. Carter, And R. Kohn (2000): "Efficient Bayesian Inference for Dynamic Mixture Models," Journal of the American Statistical Association, 95, 819-828.

Giordani, P. And R. Kohn (2008): "Efficient Bayesian Inference for Multiple Change-Point and Mixture Innovation Models," Journal of Business 8 Economic Statistics, 26, 66-77.

Hamilton, J. D. (1989): "A New Approach to the Economic Analysis of Nonstationary Time Series and the Business Cycle," Econometrica, 57, 357-384.

(1990): "Analysis of time series subject to changes in regime," Journal of Econometrics, 45, 39-70.

Heston, A., R. Summers, and B. Aten (2009): "Penn World Table Version 6.3," Center for International Comparisons of Production, Income and Prices at the University of Pennsylvania.

Kim, S., N. Shephard, And S. Chib (1998): "Stochastic Volatility: Likelihood Inference and Comparison with ARCH Models," Review of Economic Studies, 65, 361-393.

Koop, G. And S. Potter (2007): "Estimation and Forecasting in Models with Multiple Breaks," Review of Economic Studies, 74, 763-789.

(2009): "Prior Elicitation in Multiple Change-Point Models," International Economic Review, 50, 751-771.

Koopman, S. J. (1997): "Exact Initial Kalman Filtering and Smoothing for Nonstationary Time Series Models," Journal of the American Statistical Association, 92, 1630-1638.

Koopman, S. J. And J. Durbin (2002): "A Simple and Efficient Simulation Smoother for State Space Time Series Analysis," Biometrika, 89, 603-615. 
Kumar, S. And R. Russell (2002): "Technological Change, Techological Catch-up, and Capital Deepening: Relative Contributions to Growth and Covergence," American Economic Review, 92, 527-548.

Mazet, V., D. Brie, And J. Idier (2005): "Simulation of postive normal variables using several proposal distributions," 2005 IEEE/SP 13th Workshop on Statistical Signal Processing, 37-42.

Meeusen, W. And J. van den Broeck (1990): "Efficiency estimation from Cobb-Douglas production functions with composed error," International Economic Review, 8, 435-444.

Plummer, M., N. Best, K. Cowles, And K. Vines (2009): coda: Output analysis and diagnostics for $M C M C$, r package version 0.13-4.

Rodriguez-Yam, G., R. A. Davis, And L. L. Scharf (2004): "Efficient Gibbs Sampling of Truncated Multivariate Normal with Application to Constrained Linear Regression," Tech. rep., Columbia University.

Tsionas, E. G. (2006): "Inference in Dynamic Stochastic Frontier Models," Journal of Applied Econometrics, 21, 669-676.

Tsionas, E. G. And S. C. Kumbhakar (2004): "Markov switching stochastic frontier model," Econometrics Journal, 7, 398-425.

Tsionas, E. G. and K. C. Tran (2007): "Bayesian Inference in Threshold Stochastic Frontier Models," Tech. rep., Athens University of Economics and Business.

van den Broeck, J., J. Koop, Garyand Osiewalski, and M. F. Steel (1994): "Stochastic Fronier Models a Bayesian Perspective," Journal of Econometrics, 61, 273-303.

Yan, J., X. Sun, And J. J. Liu (2009): "Assessing Container Operator Efficiency with Heterogeneous and Time-Varying Production Frontiers," Transportation Research Part B, 43, 172-185.

Zellner, A. (1986): "On Assessing Prior Distributions and Bayesian Regression Analysis with G-prior Distribution," in Bayesian Inference and Decision Techniques: Essays in Honour of Bruno de Finetti, ed. by P. K. Goel and A. Zellner, Amsterdam: North-Holland, 233-243.

\section{Appendix}

\section{A Simulate draws of $\mu$ and $\phi$}

Note that $\mu$ and $\phi$ are all $M \times 1$ vectors and $M$ denotes the maximal number of in-sample regimes. It is possible that the number of in-sample regimes implied 
by $S^{\gamma}$, say $m_{\gamma}$, could be less than $M$. Hence our data can provide information for the parameters under the first $m_{\gamma}$ regimes through the posterior. For the parameters under the next $M-m_{\gamma}$ regimes, which take place outside the sample, the information from the data can only spread into them through the prior defined in equation (5). Here we just explain how to draw the parameters under the in-sample regimes. Let us first consider drawing $\phi$ conditional on $\mu$ and define $z_{j}^{\phi}=\ln \left[\sum_{i=1}^{N} \sum_{t \in\left\{t \mid S_{t}=j\right\}}\left(\gamma_{i t}-\mu_{j}\right)^{2}\right]$. Then we can have the following state space model:

$$
\begin{gathered}
z_{j}^{\phi}=\phi_{j}+e_{j}^{\phi}, \quad j=1, \ldots, m_{\gamma} \\
\phi_{j+1}=\phi_{j}+u_{j+1}^{\phi}, \quad u_{j}^{\phi} \sim N\left(0, \sigma_{\phi}^{2}\right) .
\end{gathered}
$$

Note that the exponential of $e_{j}^{\phi}$ follows a $\chi^{2}$ distribution with degrees of freedom $d_{j}^{\phi} N$, where $d_{j}^{\phi}$ is the duration of the $j$ th regime. Following Kim et al. (1998), we use a mixture normal distribution to approximate the distribution of $e_{j}^{\phi}$ and transform (14) into a partially Gaussian state space model. After we obtain a new draw, we put it through a Metropolis-Hastings (MH) step to decide whether to accept it or keep the old draw as the new draw. To approximate the distribution of $e_{j}^{\phi}$, we first generate artificial data from its distribution and then use the R package written by Fraley and Raftery (2009) to fit a mixture normal model ${ }^{9}$ to the generated data. We can achieve better approximation by increasing the sample size of the artificial data. At the end, we use $10^{6}$ as our sample size to obtain the results in the paper. Note that such approximation is a one-off process and we can save the estimation results to be used later. For different degrees of freedom, we can parallelize the estimation problem and run it on the computer cluster. To draw from the components of the mixture normal distribution, we rely on the algorithm by Carter and Kohn (1994).

Let us define $z_{j}^{* \phi}=z_{j}^{\phi}-\mu_{z^{\phi}}$, where $\mu_{z^{\phi}}$ is the mean parameter drawn from the normal mixture component conditional on $z^{\phi}-\phi$ using the algorithm by Carter and Kohn (1994). Now we are ready to simulate draws of $\sigma_{\phi}^{2}$ and $\phi$. We start the following Kalman filter by setting $a_{2 \mid 1}=z_{1}^{\phi}$ and $P_{2 \mid 1}=\sigma_{\phi}^{2}+\sigma_{z \phi}^{2}$, where $\sigma_{z^{\phi}}^{2}$ is the variance from the normal mixture. Such initial setting of the Kalman filter corresponds to the flat prior for $\phi_{1}$ in (7) (see Koopman, 1997).

$$
\begin{aligned}
& k_{j}=P_{j \mid j-1} F_{j}^{-1}, F_{j}=P_{j \mid j-1}+\sigma_{z^{\phi}}^{2}, v_{j}=z_{j}^{* \phi}-a_{j \mid j-1} \\
& a_{j+1 \mid j}=a_{j \mid j-1}+k_{j} v_{j}, P_{t+1 \mid t}=P_{t \mid t-1}\left(1-k_{t}\right)+\sigma_{\phi}^{2}, \quad \text { for } j=2, \ldots, m_{\gamma}
\end{aligned}
$$

Based on the above Kalman filter, we can integrate out $\phi$ to obtain the likelihood

\footnotetext{
${ }^{9}$ The package can estimate the number of components, the proportions of the mixture, the means and the variances of the normal distributions.
} 
function of $\sigma_{\phi}^{2}$. The posterior kernel of $\sigma_{\phi}^{2}$ takes the following form:

$$
p\left(\sigma_{\phi}^{2} \mid z^{* \phi}, \sigma_{z^{\phi}}^{2}\right) \propto p_{I G}\left(\sigma_{\phi}^{2}\right)\left(\prod_{j=2}^{m_{\gamma}} F_{j}\right) \exp \left(-\frac{1}{2} \sum_{j=2}^{m_{\gamma}} \frac{v_{j}^{2}}{F_{j}}\right) .
$$

where $p_{I G}\left(\sigma_{\phi}^{2}\right)$ is the inverted gamma prior specified in (7). The posterior distribution in (16) is not a standard distribution and we use a MH algorithm with normal distribution as the proposal density to simulate draws from it. To simulate $\phi$, we use the simulation smoother developed by De Jong and Shephard (1995) and run through the following backward recursion with $r_{m_{\gamma}}=N_{m_{\gamma}}=0$ :

$$
\begin{aligned}
& D_{j}=F_{j}^{-1}+k_{j}^{2} N_{j}, n_{j}=F_{j}^{-1} v_{j}-k_{j} r_{j}, C_{j}=\sigma_{z^{\phi}}^{2}-\sigma_{z^{\phi}}^{4} D_{j} \\
& r_{j-1}=F_{j}^{-1} v_{j}+\left(1-k_{j}\right) r_{j}-\frac{\kappa_{j} \sigma_{z^{\phi}}^{2}\left(D_{j}-k_{j} N_{j}\right)}{C_{j}} \\
& N_{j-1}=F_{j}^{-1}+\left(1-k_{j}\right)^{2} N_{j}+\frac{\sigma_{z^{\phi}}^{4}\left(D_{j}-k_{j} N_{j}\right)^{2}}{C_{j}}, \text { for } j=m_{\gamma}, \ldots, 2 .
\end{aligned}
$$

where $\left.\kappa_{j} \sim N\left(0, C_{j}\right)\right)$ and $z_{j}^{* \phi}-\sigma_{z^{\phi}}^{2} n_{j}-\kappa_{j}$ can be viewed as a signal from $\phi_{j} \mid \phi_{(j+1): m_{\gamma}}, z^{* \phi}, \sigma_{z^{\phi}}^{2}$. We set $v_{1}=z_{1}^{* \phi}, F_{1}^{-1}=0$ and $k_{1}=1$ to correspond to the flat prior for $\phi_{1}$.

To draw $\mu \mid \phi, y$ is simpler and similar to drawing $\phi \mid \mu, y$. Define $z_{j}^{\mu}=\frac{1}{d_{j}^{\mu} N} \sum_{i=1}^{N} \sum_{t \in\left\{t \mid S_{t}=j\right\}} \gamma_{i t}$, where $d_{j}^{\mu}$ the duration of the $j$ th regime. We can obtain the following state space model:

$$
\begin{aligned}
z_{j}^{\mu} & =\mu_{j}+e_{j}^{\mu}, \quad j=1, \ldots, m_{\gamma} \\
\mu_{j+1} & =\mu_{j}+u_{j+1}^{\mu}, \quad u_{j}^{\mu} \sim N\left(0, \sigma_{\mu}^{2}\right) .
\end{aligned}
$$

where $e_{j}^{\mu} \sim N\left(0, \frac{1}{d_{j}^{\mu} N} \sigma^{2}\right)$. It is obvious that (18) is just a linear Gaussian state space model. We can simply apply the simulation smoother algorithm by De Jong and Shephard (1995) to it and draw $\sigma_{\mu}^{2}$ and $\mu$ as for the case of $\phi$ conditional on the component of the mixture normal distribution.

\section{B Simulate draws of $S^{\gamma}$ and $\gamma$}

To draw $\gamma$, we numerically invert its cumulative posterior distribution function, which in turn follows a uniform distribution between 0 and 1 . Since the elements in $\gamma$ are independent of each other given other parameters, we can parallelize this computation task over the computer cluster and speed up the process. The 
posterior kernel of $\gamma_{i S_{t}=j}\left(\gamma_{i j}\right)$ takes the following form:

$$
\begin{aligned}
& p\left(\gamma_{i j} \mid S^{\gamma}, \mu, \phi, a, S^{a}, \beta, \sigma^{2}, Y\right) \propto \\
& \exp \left\{-\frac{1}{2 \sigma^{2}}\left[d_{j}\left[\exp \left(\gamma_{i j}\right)-\frac{1}{d_{j}} \sum_{t \in\left\{t \mid S_{t}=j\right\}}\left(x_{i t}^{\prime} a_{S_{t}^{a}}+w_{i t}^{\prime} \beta-y_{i t}\right)\right]^{2}\right.\right. \\
& +\sum_{t \in\left\{t \mid S_{t}=j\right\}}\left(x_{i t}^{\prime} a_{S_{t}^{a}}+w_{i t}^{\prime} \beta-y_{i t}\right)^{2} \\
& \left.\left.-\frac{1}{d_{j}}\left[\sum_{t \in\left\{t \mid S_{t}=j\right\}}\left(x_{i t}^{\prime} a_{S_{t}^{a}}+w_{i t}^{\prime} \beta-y_{i t}\right)\right]^{2}\right]\right\} \exp \left[-\frac{\left(\gamma_{i j}-\mu_{j}\right)^{2}}{2 \exp \left(\phi_{j}\right)}\right] .
\end{aligned}
$$

Tsionas (2006) and Yan et al. (2009) use MH to draw $\gamma$ from (19). We do not follow their practice here since we found that the quality of such algorithm tends to deteriorate as $N$ increases ${ }^{10}$. To have sensible posterior estimates, we have to use a large number of burn-in in the MH step.

To draw $S^{\gamma}$, we first integrate out $\gamma$ and let $S^{\gamma}$ depend on $\mu$ and $\phi$ as mentioned in the main text. Following Chib (1998), we run through the following forward recursion to find $\operatorname{Pr}\left(S_{T}^{\gamma} \mid \mu, \phi, a, S^{a}, \beta, \sigma^{2}, Y\right)^{11}$ :

$\operatorname{Pr}\left(S_{t}=j \mid \mu, \phi, y_{t}\right) \propto p\left(y_{t} \mid \mu_{j}, \phi_{j}\right) \sum_{m=j-1}^{j}\left[\operatorname{Pr}\left(S_{t}=j \mid S_{t-1}=m\right) \operatorname{Pr}\left(S_{t-1}=m \mid y_{t-1}, \mu, \phi\right)\right]$,

where $t=2, \ldots, T$ and $p\left(y_{t} \mid \mu_{j}, \phi_{j}\right)=\int p\left(y_{t} \mid \gamma_{j}\right) p\left(\gamma_{j} \mid \mu_{j}, \phi_{j}\right) d \gamma_{j}$ and $\operatorname{Pr}\left(S_{1}=\right.$ $\left.1 \mid y_{1}\right)=1$. When we finish the above recursion, we can draw $S_{T}^{\gamma} \mid Y, \mu, \phi$. To draw the regime states at other periods, the following backward recursion given by Chib (1998) is used.

$$
\operatorname{Pr}\left(S_{t} \mid Y, S_{(t+1): T}, \mu, \phi\right) \propto \operatorname{Pr}\left(S_{t} \mid y_{t}, \mu, \phi\right) \operatorname{Pr}\left(S_{t+1} \mid S_{t}\right), \quad \text { for } t=T-1, \ldots, 2 .
$$

\section{Simulate draws of $S^{a}$ and $a$}

To simulate $a \mid S^{a}$ given $S^{a}$ and other parameters is similar to drawing $\mu$ before. However, drawing and smoothing the initial state requires special attention since we are using a diffuse prior for $a$. Define

$$
\begin{aligned}
z_{i j}^{a} & =\frac{1}{d_{j}^{a}} \sum_{t \in\left\{t \mid S_{t}^{a}=j\right\}}\left[y_{i t}-w_{i t}^{\prime} \beta+\exp \left(\gamma_{i S_{t}^{\gamma}}\right)\right] \\
\zeta_{j} & =\frac{1}{d_{j}^{a}} \sum_{t \in\left\{t \mid S_{t}^{a}=j\right\}} x_{i t}
\end{aligned}
$$

\footnotetext{
${ }^{10}$ The results are available upon request from the author.

${ }^{11}$ For simplicity, we suppress the reliance on $a, S^{a}, \beta$ and $\sigma^{2}$ for the moment.
} 
where $d_{j}^{a}$ is the duration of the $j$ th regime for $a$. Denote $z_{j}^{a}=\left(z_{1 j}^{a}, z_{1 j}^{a}, \ldots, z_{N j}^{a}\right)^{\prime}$ and $\Xi_{j}=\left(\zeta_{1 j}, \zeta_{1 j}, \ldots, \zeta_{N j}\right)^{\prime}$. Then we can have the following state space model:

$$
\begin{array}{ll}
z_{j}^{a}=\Xi_{j} a_{j}+e_{j}^{a}, & e_{j}^{a} \sim N\left(0, \frac{\sigma^{2}}{d_{j}^{a}} I_{N}\right), \quad, \text { for } j=1, \ldots, m_{a} \\
a_{j+1}=a_{j}+u_{j}^{a}, & u_{j}^{a} \sim N\left(0, \sigma_{a}^{2} I\right)
\end{array}
$$

where $m_{a}$ is the number of in-sample regimes for $a$. Due to the diffuse prior for $a_{1}$, the Kalman Filter now takes the following form (see Koopman, 1997):

$$
\begin{aligned}
& R_{j}^{*}=\Xi_{j}\left(V_{j-1}^{*}+\sigma_{a}^{2} I\right) \Xi_{j}^{\prime}+\frac{\sigma^{2}}{d_{j}^{a}} I_{N}, R_{j}^{\infty}=\Xi_{j} V_{j-1}^{\infty} \Xi_{j}^{\prime}, \\
& J_{j}^{*}=\left(V_{j-1}^{*}+\sigma_{a}^{2} I\right) \Xi_{j}^{\prime}, J_{j}^{\infty}=V_{j-1}^{\infty} \Xi_{j}^{\prime} ; \\
& \text { if } \operatorname{rank}\left(R_{j}^{\infty}\right)=N, M_{j}=J_{j}^{\infty}\left(R_{j}^{\infty}\right)^{-1}, C_{j}^{\infty}=J_{j}^{\infty}\left(R_{j}^{\infty}\right)^{-1} J_{j}^{\infty^{\prime}}, \\
& \quad C_{j}^{*}=J_{j}^{*}\left(R_{j}^{\infty}\right)^{-1} J_{j}^{\infty}+J_{j}^{\infty}\left(R_{j}^{\infty}\right)^{-1}\left(J_{j}^{*}-J_{j}^{\infty}\left(R_{j}^{\infty}\right)^{-1} R_{j}^{*}\right)^{\prime} ; \\
& \text { if } \operatorname{rank}\left(R_{j}^{\infty}\right)<N, M_{j}=J_{j}^{*} G_{2 j} G_{2 j}^{\prime}+J_{j}^{\infty} G_{1 j} G_{1 j}^{\prime}, C_{j}^{\infty}=J_{j}^{\infty} G_{1 j} G_{1 j}^{\prime} J_{j}^{\prime}, \\
& \quad C_{j}^{*}=J_{j}^{*} M_{j}^{\prime}+J_{j}^{\infty} G_{1 j} G_{1 j}^{\prime}\left(J_{j}^{*}-J_{j}^{\infty} G_{1 j} G_{1 j}^{\prime} R_{j}^{*}\right)^{\prime} ; \\
& \text { if } \operatorname{rank}\left(R_{j}^{\infty}\right)=0, M_{j}=J_{j}^{*}\left(R_{j}^{*}\right)^{-1}, C_{j}^{*}=J_{j}^{*}\left(R_{j}^{*}\right)^{-1} J_{j}^{\prime}{ }^{*}, C_{j}^{\infty}=0 ; \\
& V_{j}^{*}=V_{j-1}^{*}+\sigma_{a}^{2} I-C_{j}^{*}, V_{j}^{\infty}=V_{j-1}^{\infty}-C_{j}^{\infty}, m_{j}=m_{j-1}+M_{j}\left(z_{j}^{a}-\Xi_{j} a_{j}\right) .
\end{aligned}
$$

According to Koopman (1997), we can find two matrices $G_{1 j}$ and $G_{2 j}$ such that $\left(G_{1 j}, G_{2 j}\right)$ is a full rank matrix, $\left(G_{1 j}, G_{2 j}\right)^{\prime} R_{j}^{\infty}\left(G_{1 j}, G_{2 j}\right)=\left(\begin{array}{cc}I_{r} & 0 \\ 0 & 0\end{array}\right)$ and $\left(G_{1 j}, G_{2 j}\right)^{\prime} R_{j}^{*}\left(G_{1 j}, G_{2 j}\right)=\left(\begin{array}{cc}L_{j}^{*} & 0 \\ 0 & I_{N-r}\end{array}\right)$, where $r$ is the rank of $R_{j}^{\infty}$ and $\operatorname{rank}\left(L_{j}^{*}\right) \leq r$. Suppose the dimension of $a$ is $k_{a}$. We initialize the filter by setting $m_{0}=0_{k_{a}}, V_{0}^{\infty}=I_{k_{a}}$ and $V_{0}^{*}+\sigma_{a}^{2} I=0$, which is consistent with the flat prior for $a_{1}$ in (7). In comparison to the ordinary Kalman filter, (24) computes extra quantities: $V_{j}^{\infty}, R_{j}^{\infty}, C_{j}^{\infty}$ and $J_{j}^{\infty}$. Koopman (1997) showed that $\operatorname{rank}\left(V_{j+1}^{\infty}\right) \leq \operatorname{rank}\left(V_{j}^{\infty}\right)-\operatorname{rank}\left(R_{j+1}^{\infty}\right)$, which means $V_{1}^{\infty}=0$ given that $\Xi_{j}$ has full colum rank. Hence from $j=2$, (24) becomes ordinary Kalman filter. The filter in (24) poses some computational difficulties when $N$ is a large number. We can parallelize matrix multiplication for faster speed. To invert $\left(R_{j}^{*}\right)^{-1}$, the following identity is used:

$$
\left(R_{j}^{*}\right)^{-1}=\frac{d_{j}^{a}}{\sigma^{2}}\left\{I_{N}-\Xi_{j}\left[\frac{\sigma^{2}}{d_{j}^{a}}\left(V_{t-1}^{*}+\sigma_{a}^{2} I\right)^{-1}+\Xi_{j}^{\prime} \Xi_{j}\right]^{-1} \Xi_{j}^{\prime}\right\}
$$

So instead of inverting an $N \times N$ matrix, we now just need to invert two $k_{a} \times k_{a}$ matrices.

To draw $a \mid z^{a}$ (smoothing), we use the algorithm developed by Koopman and Durbin (2002), which is more efficient than De Jong and Shephard (1995) 
especially when $a$ is not a scalar. We run the following backward recursion with $r_{m_{a}}=0$ after the Kalman filter in (24) completes:

$$
\begin{aligned}
& r_{j-1}=\Xi_{j}^{\prime} R_{j}^{-1}\left(z_{j}^{a}-\Xi_{j} m_{j-1}\right)+\left(I-M_{j} \Xi_{j}\right)^{\prime} r_{j}, \hat{u}_{j}=\sigma_{a}^{2} r_{j} \\
& \hat{e}_{j}^{a}=\frac{\sigma^{2}}{d_{j}^{a}}\left[R_{j}^{-1}\left(z_{j}^{a}-\Xi_{j} m_{j-1}\right)-M_{j}^{\prime} r_{j}\right]
\end{aligned}
$$

where $R_{j}^{-1}=0$ for $j=1$ and $R_{j}=R_{j}^{*}$ for $j=2, \ldots, m_{a}$ if $\Xi_{j}$ has full colum rank. We generate $u_{j}^{a+}$ and $e_{j}^{a+}$ from their respective distributions in (23) and hence $z_{j}^{a+}$ and $a_{j}^{+}$with $a_{1}^{+}=0$. We then calculate $\hat{u}_{j}^{a+}$ and $\hat{e}_{j}^{a+}$ from (26) with $z_{j}^{a}$ and $a_{j}$ replaced by $z_{j}^{a+}$ and $a_{j}^{+}$in (24) and (26). The smoothed errors are obtained as $\tilde{e}_{j}^{a}=\hat{e}_{j}^{a}+e_{j}^{a+}-\hat{e}_{j}^{a+}$ and $\tilde{u}_{j}^{a}=\hat{u}_{j}^{a}+u_{j}^{a+}-\hat{u}_{j}^{a+}$. To generate the smoothed state $\tilde{a}_{j}$, we can use $(23)$ once $\tilde{a}_{1}$ is found and $\tilde{a}_{1}=\left(\Xi_{1}^{\prime} \Xi_{1}\right)^{-1} \Xi_{1}^{\prime}\left(z_{1}^{a}-\tilde{e}_{1}^{a}\right)$.

To draw $S^{a}$, we use the algorithm by Gerlach et al. (2000) and let $S^{a}$ be unconditional on $a$. Define $z_{i t}^{* a}=\left[y_{i t}-w_{i t}^{\prime} \beta+\exp \left(\gamma_{i S_{t}^{\gamma}}\right)\right], z_{t}^{* a}=\left(z_{1 t}^{* a}, z_{2 t}^{* a}, \ldots, z_{N t}^{* a}\right)^{\prime}$ and $\Xi_{t}^{*}=\left(x_{1 t}, x_{2 t}, \ldots, x_{N t}\right)^{\prime}$. We then write down the following state space model:

$$
\begin{aligned}
z_{t}^{* a} & =\Xi_{t}^{*} a_{t}+e_{t}^{* a}, & e_{t}^{* a} & \sim N\left(0, \sigma^{2} I_{N}\right) \\
a_{t+1} & =a_{t}+K_{t} u_{t}, & u_{t} & \sim i . i . d . N\left(0, \sigma_{a}^{2} I\right) .
\end{aligned}
$$

where $K_{t}=S_{t+1}-S_{t}$ takes the value of either 1 or 0 indicating whether period $t$ is a break point or not. We draw the posterior of $K^{a}=\left(K_{1}, \ldots, K_{T-1}\right)^{\prime}$ and then transform $K^{a}$ into $S^{a}=\left(S_{1}, \ldots, S_{T}\right)^{\prime}$. We still use the prior proposed by Koop and Potter (2009) and represent it as (12). The algorithm by Gerlach et al. (2000) is a Gibbs sampler, which draws $K_{t} \mid K_{s \neq t}, Y$, and the details are as follow.

Algorithm C.1. 1. Given the initial draw of $K^{a}$, we run through the following backward recursion, where the notations are just confined to this section.

$$
\begin{aligned}
& N_{t+1}^{-1}=\left[\sigma_{a}^{2} K_{t} \Xi_{t+1}^{*}\left(\Xi_{t+1}^{*}\right)^{\prime}+\sigma^{2} I_{N}\right]^{-1}=\frac{1}{\sigma^{2}}\left[I_{N}-\Xi_{t+1}^{*}\left(\frac{\sigma^{2}}{\sigma_{a}^{2} K_{t}} I+\left(\Xi_{t+1}^{*}\right)^{\prime} \Xi_{t+1}^{*}\right)^{-1}\left(\Xi_{t+1}^{*}\right)^{\prime}\right], \\
& B_{t+1}=\sigma_{a}^{2} K_{t}\left(\Xi_{t+1}^{*}\right)^{\prime} N_{t+1}^{-1}, A_{t+1}=I_{k_{a}}-B_{t+1} \Xi_{t+1}^{*} \\
& Z_{t+1} Z_{t+1}^{\prime}=\sigma_{a}^{a} K_{t} A_{t+1} A_{t+1}^{\prime}+\sigma^{2} B_{t+1} B_{t+1}^{\prime}, D_{t+1}=Z_{t+1}^{\prime} \Omega_{t+1} Z_{t+1}+I \\
& \Omega_{t}=A_{t+1}^{\prime}\left(\Omega_{t+1}-\Omega_{t+1} Z_{t+1} D_{t+1}^{-1} Z_{t+1}^{\prime} \Omega_{t+1}\right) A_{t+1}+\left(\Xi_{t+1}^{*}\right)^{\prime} N_{t+1}^{-1} \Xi_{t+1}^{*}, \\
& \mu_{t}=A_{t+1}^{\prime}\left(I-\Omega_{t+1} Z_{t+1} D_{t+1}^{-1} Z_{t+1}^{\prime}\right)\left(\mu_{t+1}-\Omega_{t+1} B_{t+1} z_{t+1}^{* a}\right)+\left(\Xi_{t+1}^{*}\right)^{\prime} N_{t+1}^{-1} z_{t+1}^{* a}, t=T-1, \ldots, 1 .
\end{aligned}
$$

where $\Omega_{T}=0_{k_{a} \times k_{a}}, \mu_{T}=0_{k_{a} \times 1}$ and $Z_{t+1}$ either has full column rank or is null.

2. For $K_{t}=0,1$, 
(a) run the Kalman filter similar to (24) by replacing the subscript $j$ by $t(t=1, \ldots, T-1), \sigma_{a}^{2}$ by $K_{t} \sigma_{a}^{2}, \frac{\sigma^{2}}{d_{j}^{a}}$ by $\sigma^{2}, \Xi$ by $\Xi^{*}$ and $z^{a}$ by $z^{* a}$.

(b) Calculate the posterior conditional:

$$
\begin{aligned}
& \operatorname{Pr}\left(K_{t} \mid z^{* a}, K_{s \neq t}\right) \propto p\left(z_{t+1}^{* a} \mid z_{1: t}^{* a}, K_{1: t}\right) p\left(z_{(t+2): T}^{* a} \mid z_{1:(t+1)}, K^{a}\right) \operatorname{Pr}\left(K_{t} \mid K_{s \neq t}\right) \\
& p\left(z_{t+1}^{* a} \mid z_{1: t}^{* a}, K_{1: t}\right) \propto\left|R_{t+1}\right|^{-\frac{1}{2}} \exp \left[-\frac{1}{2}\left(z_{t+1}^{* a}-\Xi_{t+1}^{*} m_{t}\right)^{\prime} R_{t+1}^{-1}\left(z_{t+1}^{* a}-\Xi_{t+1}^{*} m_{t}\right)\right] \\
& \quad p\left(z_{(t+2): T}^{* a} \mid z_{1:(t+1)}, K^{a}\right) \propto\left|T_{t+1}^{\prime} \Omega_{t+1} T_{t+1}+I\right|^{-\frac{1}{2}} \\
& \quad \quad \exp \left\{-\frac{1}{2}\left[m_{t+1}^{\prime} \Omega_{t+1} m_{t+1}-2 \mu_{t+1}^{\prime} m_{t+1}-\phi_{t+1}^{\prime}\left(T_{t+1}^{\prime} \Omega_{t+1} T_{t+1}+I\right)^{-1} \phi_{t+1}\right]\right\} \\
& V_{t+1}=T_{t+1} T_{t+1}^{\prime}, \phi_{t+1}=T_{t+1}^{\prime}\left(\mu_{t+1}-\Omega_{t+1} m_{t+1}\right)
\end{aligned}
$$

where $\operatorname{Pr}\left(K_{t} \mid K_{s \neq t}\right)$ is obtained from the prior and $T_{t+1}$ either has full column rank or is null. The determinant of $R$ is calculated as:

$$
\left|R_{t+1}\right|=\left(\sigma^{2}\right)^{N-k_{a}}\left|V_{t}+\sigma_{a}^{2} K_{t}\right|\left|\sigma^{2}\left(V_{t}+\sigma_{a}^{2} K_{t}\right)^{-1}+\left(\Xi_{t+1}^{*}\right)^{\prime} \Xi_{t+1}^{*}\right|
$$

(c) Draw $K_{t} \mid z^{* a}, K_{s \neq t}$ based on the results from (29) and use the values of $m_{t+1}$ and $V_{t+1}$ accordingly for next iteration.

\section{Simulate draws of $\sigma^{2}$ and $\beta$}

It is straightforward to draw $\sigma^{2}$ from inverted gamma distribution. The posterior of $\beta \mid \sigma^{2}, a, \gamma, Y$ is a vector normal distribution truncated to the positive region. We use the algorithm proposed by Rodriguez-Yam et al. (2004) for this purpose. This algorithm involves drawing from scalar truncated normal distribution, which is done through the algorithm by Mazet et al. (2005). 